\title{
Ergodicity for multidimensional jump diffusions with position dependent jump rate
}

\author{
Eva Löcherbach ${ }^{\mathrm{a}}$ and Victor Rabiet ${ }^{\mathrm{b}}$ \\ ${ }^{a}$ CNRS UMR 8088, Département de Mathématiques, Université de Cergy-Pontoise, France. E-mail: eva.loecherbach@u-cergy.fr \\ b LAMA - Laboratoire d'Analyse et de Mathématiques Appliquées, France. E-mail: victor.rabiet@ens-cachan.fr
}

Received 21 April 2015; revised 1 March 2016; accepted 1 March 2016

Abstract. We consider a jump type diffusion $X=\left(X_{t}\right)_{t}$ with infinitesimal generator given by

$$
L \psi(x)=\frac{1}{2} \sum_{1 \leq i, j \leq d} a_{i j}(x) \frac{\partial^{2} \psi(x)}{\partial x_{i} \partial x_{j}}+g(x) \nabla \psi(x)+\int_{\mathbb{R}^{d}}(\psi(x+c(z, x))-\psi(x)) \gamma(z, x) \mu(\mathrm{d} z),
$$

where $\mu$ is of infinite total mass. We prove Harris recurrence of $X$ using a regeneration scheme which is entirely based on the jumps of the process. Moreover we state explicit conditions in terms of the coefficients of the process allowing to control the speed of convergence to equilibrium in terms of deviation inequalities for integrable additive functionals.

Résumé. On considère une diffusion $X=\left(X_{t}\right)_{t}$, avec des sauts, correspondant au générateur infinitésimal suivant :

$$
L \psi(x)=\frac{1}{2} \sum_{1 \leq i, j \leq d} a_{i j}(x) \frac{\partial^{2} \psi(x)}{\partial x_{i} \partial x_{j}}+g(x) \nabla \psi(x)+\int_{\mathbb{R}^{d}}(\psi(x+c(z, x))-\psi(x)) \gamma(z, x) \mu(\mathrm{d} z)
$$

où $\mu$ est de masse totale infinie. On prouve ici la récurrence au sens de Harris de $X$ en utilisant un schéma de régénération entièrement basé sur les sauts du processus. De plus, on donnera des conditions explicites en terme de coefficients du processus $X$ permettant de contrôler la vitesse de convergence à l'équilibre en terme d'inégalités de déviations pour des fonctionnelles additives intégrables.

MSC: 60J55; 60J35; 60F10; 62M05

Keywords: Diffusions with jumps; Harris recurrence; Nummelin splitting; Continuous time Markov processes; Additive functionals

\section{Introduction}

Let $N(\mathrm{~d} s, \mathrm{~d} z, \mathrm{~d} u)$ be a Poisson random measure on $\mathbb{R}_{+} \times \mathbb{R}^{d} \times \mathbb{R}_{+}$, defined on a probability space $(\Omega, \mathcal{A}, P)$ with intensity measure $\mathrm{d} s \mu(\mathrm{d} z) \mathrm{d} u$, where $\mu$ is a $\sigma$-finite measure on $\mathbb{R}^{d}$. We consider a process $X=\left(X_{t}\right)_{t \geq 0}, X_{t} \in \mathbb{R}^{d}$, solution of

$$
X_{t}=x+\int_{0}^{t} g\left(X_{s}\right) \mathrm{d} s+\int_{0}^{t} \sigma\left(X_{s}\right) \mathrm{d} W_{s}+\int_{[0, t]} \int_{\mathbb{R}^{d} \times \mathbb{R}_{+}} c\left(z, X_{s-}\right) 1_{u \leq \gamma\left(z, X_{s-}\right)} N(\mathrm{~d} s, \mathrm{~d} z, \mathrm{~d} u),
$$


$x \in \mathbb{R}^{d}$, where $W$ is an $m$-dimensional Brownian motion. The associated infinitesimal generator is given for smooth test functions by

$$
L \psi(x)=\frac{1}{2} \sum_{1 \leq i, j \leq d} a_{i j}(x) \frac{\partial^{2}}{\partial x_{i} \partial x_{j}} \psi(x)+g(x) \nabla \psi(x)+\int_{\mathbb{R}^{d}}(\psi(x+c(z, x))-\psi(x)) \gamma(z, x) \mu(\mathrm{d} z),
$$

where $a=\sigma \sigma^{*}$. Notice that the jump rate at time $t$ of process depends on the position of the process $X_{t}$ itself, i.e. the intensity measure in the infinitesimal operator $L$ is $\gamma(z, x) \mu(\mathrm{d} z)$. We will suppose that $\int \gamma(z, x) \mu(\mathrm{d} z)=\infty$, i.e., jumps occur with infinite activity and the process possesses infinitely many small jumps during any finite time interval $[0, T]$.

The principal aim of the present paper is to give easily verifiable conditions on the coefficients $g, \sigma, c$ and $\gamma$ under which the process is recurrent in the sense of Harris and satisfies the ergodic theorem, without imposing any nondegeneracy condition on the diffusive part. Recall that a process $X$ is called recurrent in the sense of Harris if it possesses an invariant measure $m$ such that any set $A$ with $m(A)>0$ is visited infinitely often almost surely (see Azéma, Duflo and Revuz [2] (1969)): For all $x \in \mathbb{R}^{d}$,

$$
P_{x}\left[\int_{0}^{\infty} 1_{A}\left(X_{S}\right) \mathrm{d} s=\infty\right]=1
$$

For classical jump diffusions there starts to be a huge literature on this subject, see e.g. Masuda [9] (2007) who works in a simpler situation where the intensity term $1_{u \leq \gamma\left(z, X_{s-}\right)}$ is not present. In order to prove recurrence, Masuda follows the Meyn and Tweedie approach developed in [10] or [11]. Kulik [7] (2009) uses the stratification method in order to prove exponential ergodicity of jump diffusions, but the models he considers do not include the situation with a position dependent jump rate neither. Finally, let us mention Duan and Qiao [4] (2014) who are interested in solutions driven by non-Lipschitz coefficients.

On the contrary to the above mentioned papers, in our model, jumps occur at a given intensity depending both on the current position of the process and on an additional variable $z$ chosen according to $\mu(\mathrm{d} z)$. The presence of this intensity term $\gamma\left(z, X_{s-}\right)$ in (1.1) is in fact quite natural, but it implies that the study of $X$ is technically more involved than the easier situation when $\gamma$ is lower-bounded and strictly positive.

The aim of the present paper is to show that the jumps themselves can be used in order to generate a splitting scheme which implies the recurrence of the process. The method we use is the so-called regeneration method which we apply to the big jumps. More precisely, for some suitable set $E$ such that $\mu(E)<\infty$, we cut the trajectory of $X$ into excursions between successive jumps appearing due to choices of $z$ in $E$. In spirit of the splitting technique introduced by Nummelin [12] (1978) and Athreya and Ney [1] (1978), we state a non-degeneracy condition which guarantees that the jump operator associated to the big jumps possesses a Lebesgue absolutely continuous component. This amounts to imposing that the partial derivatives of the jump term $c$ with respect to $z$ are sufficiently non-degenerate, see (2.6) and (2.7) below. We stress that we do not need any non-degeneracy condition for the diffusion coefficient $\sigma$.

In this situation we will be able to construct a sequence of regeneration times $R_{n}$ such that the trajectories $\left(X_{\left(R_{n}+t\right), t<R_{n+1}-R_{n}}\right)_{n \geq 1}$ are i.i.d. In particular, 'regeneration generates independence immediately, i.e. at each regeneration time $R_{n}$, the 'future' $X_{R_{n}+t}, t \geq 0$ is independent of the past $\sigma\left\{X_{s}, s<R_{n}\right\}$, without imposing any time lag as usual in the study of processes in continuous time.

Notice that we do not apply the splitting technique to an extracted sampled chain nor to the resolvent chain as in Meyn and Tweedie [11] (1993); the loss of memory needed for regeneration is produced only by big jumps. This approach is very natural in this context, but does not seem to be used so far in the literature, except for Xu [15] (2011), who works in a very specific frame where the jumps do not depend on the position of the process.

Our paper is organized as follows. In Section 2 we state our main assumptions, prove a lower bound which is of local Doeblin type and state our main results on Harris recurrence and speed of convergence to equilibrium of the process. Section 3 introduces the regeneration technique based on big jumps and proves the existence of certain (polynomial) moments of the associated regeneration times. Section 4 is devoted to an informal discussion on explicit and easily verifiable conditions stated in terms of the coefficients $g, \sigma, c$ and $\gamma$ which imply the Harris recurrence. Finally, we give in Section 5 a proof of the local Doeblin condition. 


\section{Basic assumptions and main results}

Consider a Poisson random measure $N(\mathrm{~d} s, \mathrm{~d} z, \mathrm{~d} u)$ on $\mathbb{R}_{+} \times \mathbb{R}^{d} \times \mathbb{R}_{+}$, defined on a probability space $(\Omega, \mathcal{A}, P)$, with intensity measure $\mathrm{d} s \mu(\mathrm{d} z) \mathrm{d} u$, where $\mu$ is a $\sigma$-finite measure on $\left(\mathbb{R}^{d}, \mathcal{B}\left(\mathbb{R}^{d}\right)\right)$. Let $X=\left(X_{t}\right)_{t \geq 0}, X_{t} \in \mathbb{R}^{d}$, be a solution of

$$
X_{t}=x+\int_{0}^{t} g\left(X_{s}\right) \mathrm{d} s+\int_{0}^{t} \sigma\left(X_{s}\right) \mathrm{d} W_{s}+\int_{[0, t]} \int_{\mathbb{R}^{d} \times \mathbb{R}_{+}} c\left(z, X_{s-}\right) 1_{u \leq \gamma\left(z, X_{s-}\right)} N(\mathrm{~d} s, \mathrm{~d} z, \mathrm{~d} u),
$$

$x \in \mathbb{R}^{d}$, where $W$ is an $m$-dimensional Brownian motion, $m \geq 1$. Write $\mathbb{F}=\left(\mathcal{F}_{t}\right)_{t \geq 0}$ for the canonical filtration of the process given by

$$
\mathcal{F}_{t}=\sigma\left\{W_{s}, N([0, s] \times A \times B), s \leq t, A \in \mathcal{B}\left(\mathbb{R}^{d}\right), B \in \mathcal{B}\left(\mathbb{R}_{+}\right)\right\} .
$$

Throughout this paper, for any $x \in \mathbb{R}^{d},|x|$ will denote the Euclidean norm on $\mathbb{R}^{d}$. Moreover, for $d \times d$ matrices $A$, $\|A\|$ denotes the associated operator norm.

\subsection{Existence of the process and non-degeneracy of the jump measure}

In order to grant existence and uniqueness of the above equation, throughout this article, we impose the following conditions on the coefficients $g, \sigma, c$ and $\gamma$.

\section{Assumption 2.1.}

1. $g$ and $\sigma$ are globally Lipschitz continuous.

2. $c$ and $\gamma$ are Lipschitz continuous with respect to $x$, i.e.

$$
\left|c(z, x)-c\left(z, x^{\prime}\right)\right| \leq L_{c}(z)\left|x-x^{\prime}\right| \quad \text { and } \quad\left|\gamma(z, x)-\gamma\left(z, x^{\prime}\right)\right| \leq L_{\gamma}(z)\left|x-x^{\prime}\right|,
$$

where $L_{c}, L_{\gamma}$ are functions $\mathbb{R}^{d} \rightarrow \mathbb{R}_{+}$.

3. $\sup _{x} \int_{\mathbb{R}^{d}}\left(L_{c}(z) \gamma(z, x)+L_{\gamma}(z)|c(z, x)|\right) \mu(\mathrm{d} z)<\infty$.

4. $\sup _{x} \int_{\mathbb{R}^{d}} \gamma(z, x)|c(z, x)| \mu(\mathrm{d} z)<\infty$.

Under these assumptions, Theorem 1.2. of Graham [5] (1992) implies that (2.1) admits a unique strong nonexplosive adapted solution which is Markov, having càdlàg trajectories.

Notice that our assumptions do not imply that there exists a finite total jump rate

$$
\int_{\mathbb{R}^{d}} \gamma(z, x) \mu(\mathrm{d} z)
$$

for any $x \in \mathbb{R}^{d}$. In other words, the above integral might be equal to $+\infty$, and jumps occur with infinite activity. We also stress that due to the presence of the intensity term $1_{u \leq \gamma\left(z, X_{s-}\right)}$ in equation (2.1) we are not in the classical frame of jump diffusions where the jump term depends in a smooth manner on $z$ and $x$.

In the present article we are seeking for conditions ensuring that the process $X$ is recurrent in the sense of Harris without using additional regularity of the coefficients, based on some minimal non-degeneracy of the jumps and without imposing any non-degeneracy condition on $\sigma$. Recall that a process $X$ is called recurrent in the sense of Harris if it possesses an invariant measure $m$ such that any set $A$ of positive $m$-measure $m(A)>0$ is visited infinitely often by the process almost surely (see Azéma, Duflo and Revuz [2] (1969)): For all $x \in \mathbb{R}^{d}$,

$$
P_{x}\left[\int_{0}^{\infty} 1_{A}\left(X_{S}\right) \mathrm{d} s=\infty\right]=1
$$

We will prove Harris recurrence by introducing a splitting scheme that is entirely based on the big jumps of $X$. In order to do so, we introduce the following additional assumption which is a non-degeneracy condition on the jump noise and the associated jump rate. 
Assumption 2.2. Writing the Lebesgue decomposition $\mu=\mu_{a c}+\mu_{s}$, with $\mu_{a c}(\mathrm{~d} z)=h(z) \mathrm{d} z$, for some measurable function $h \geq 0 \in \mathrm{L}_{\text {loc }}^{1}(\lambda)$, $\lambda$ the Lebesgue measure on $\mathbb{R}^{d}$, we suppose that there exist $x_{0}, z_{0} \in \mathbb{R}^{d}$ and $r, R>0$ such that

$$
\inf _{z:\left|z-z_{0}\right| \leq R, x:\left|x-x_{0}\right| \leq r} \gamma(z, x) h(z)=\varepsilon>0 .
$$

In the following we explain what we mean by big jumps.

\subsection{Coupling with a dominating Poisson process}

In order to introduce what we shall call big jumps, we impose the following condition which implies that the measure $\gamma(x, z) \mu(\mathrm{d} z)$ is sigma-finite, uniformly in $x$.

Assumption 2.3. There exists a non-decreasing sequence $\left(E_{n}\right)_{n}$ of subsets of $\mathbb{R}^{d}$ and an increasing sequence of positive numbers $\Gamma_{n}$ with $\Gamma_{n} \uparrow+\infty$ as $n \rightarrow \infty$, such that $\bigcup E_{n}=\mathbb{R}^{d}$,

$$
\int_{E_{n}} \gamma(z, x) \mu(\mathrm{d} z)=: \bar{\gamma}_{n}(x) \leq \Gamma_{n}<\infty
$$

for all $n$.

We fix some $n$. Thanks to (2.2), we can couple the process $X_{t}$ with a rate $\Gamma_{n}$-Poisson process $N^{[n]}=\left(N_{t}^{[n]}\right)_{t \geq 0}$ such that jumps of $X_{t}$ produced by noise $z \in E_{n}$,

$$
\Delta X_{t}=\int_{E_{n}} \int_{0}^{\infty} c\left(z, X_{t-}\right) 1_{\left\{u \leq \gamma\left(z, X_{t-}\right)\right\}} N(\mathrm{~d} t, \mathrm{~d} z, \mathrm{~d} u),
$$

can only occur at the jump times $T_{k}^{[n]}, k \geq 1$, of $N^{[n]}$. We will construct our regeneration scheme based on these big jumps $T_{k}^{[n]}, k \geq 1$, for a suitably chosen truncation level $n$.

Let

$$
\Pi(x, \mathrm{~d} y)=\mathcal{L}\left(X_{T_{k}^{[n]}} \mid X_{T_{k}^{[n]}-}=x\right)(\mathrm{d} y)
$$

be the associated transition kernel. Our aim is to obtain a local Doeblin condition of the type

$$
\Pi(x, \mathrm{~d} y) \geq 1_{C}(x) \beta v(\mathrm{~d} y),
$$

for a suitable measurable set $C$, some $\beta \in] 0,1[$ and a suitable probability measure $v$.

First notice that $\Gamma_{n}$ in (2.2) is only an upper bound on the total jump rate produced by noise belonging to $E_{n}$. As a consequence, for any $k \geq 1$ and on the event that $X_{T_{k}^{[n]}-}=x$, jumps are only accepted with probability $\frac{\bar{\gamma}_{n}(x)}{\Gamma_{n}}$. Moreover, it is easy to see that the following lower bound holds. Write $\mathcal{K}=\left\{x \in \mathbb{R}^{d}:\left|z-z_{0}\right| \leq R\right\}$ with $z_{0}$ and $R$ chosen according to Assumption 2.2. Then for all $V \in \mathcal{B}\left(\mathbb{R}^{d}\right)$,

$$
\begin{aligned}
\Pi(x, V) & \geq \frac{1}{\Gamma_{n}} \int_{E_{n} \cap \mathcal{K}} \gamma(z, x) 1_{V}(x+c(z, x)) \mu(\mathrm{d} z) \\
& \geq \frac{1}{\Gamma_{n}} \int_{E_{n} \cap \mathcal{K}} \gamma(z, x) 1_{V}(x+c(z, x)) h(z) \mathrm{d} z,
\end{aligned}
$$

where $h$ is the Lebesgue density of the absolute continuous part of $\mu$. It is natural to use a change of variables in the r.h.s. of the above lower bound, i.e. to replace, for fixed initial position $x$, the argument $x+c(x, z)$ by $y=y(z)$, on suitable subsets of $\mathbb{R}^{d}$ where $z \mapsto x+c(x, z)$ is a diffeomorphism. The difficulty is to control the dependence on the starting point $x$, since we are seeking for uniform lower bounds (2.4), uniform in $x \in C$. 
To be able to achieve such a control we impose the following regularity condition on the jump height $c(z, x)$ which implies that $z \mapsto c(z, x)$ is a local diffeomorphism, uniformly with respect to $x$ where $x$ is allowed to vary in some small balls.

Assumption 2.4. Let $x_{0}, z_{0} \in \mathbb{R}^{d}$ and $r, R>0$ as in Assumption 2.2.

1. We assume that for all $x \in \mathbb{R}^{d}$ with $\left|x-x_{0}\right| \leq r$, there exists $A>0$ with

$$
\left|\nabla_{z} c\left(z_{0}, x\right) y\right| \geq A|y|, \quad \forall y \in \mathbb{R}^{d} .
$$

2. There exists $K>0$ such that for all $x, z \in \mathbb{R}^{d}$ with $\left|z-z_{0}\right| \leq R$ and $\left|x-x_{0}\right| \leq r$,

$$
\left\|\left(\nabla_{z} c\left(z_{0}, x\right)\right)^{-1}\right\| \sum_{i, j}\left|\frac{\partial^{2} c}{\partial z_{i} \partial z_{j}}(z, x)\right| \leq \frac{K}{d} .
$$

3. $S=\sup _{z:\left|z-z_{0}\right| \leq R} \sup _{x:\left|x-x_{0}\right| \leq r} \sup _{i}\left|\partial_{z_{i}} c(z, x)\right|<\infty$,

where $h(z)$ is the Lebesgue density of the absolutely continuous part of $\mu$.

Remark 2.5. Let us explain briefly the heuristic of the preceding assumptions. Our main objective is to obtain a lower bound of the type

$$
\inf _{x:\left|x-x_{0}\right|<\eta} P\left[X_{T_{k}^{[n]}} \in V \mid X_{T_{k}^{[n]}-}=x\right]=\inf _{x:\left|x-x_{0}\right|<\eta} \Pi(x, V) \geq c \lambda(V \cap B)
$$

(compare to (2.9) below), where $c$ is some constant, $\lambda$ the Lebesgue measure on $\mathbb{R}^{d}$ and where the radius $\eta$ has to be determined. Since we have (2.5), we need to find $B\left(z_{0}, R\right)$ and $B\left(x_{0}, \eta\right)$ such that there exists a ball $B$ verifying

$$
B \subset \Psi_{x}\left(B\left(z_{0}, R\right)\right), \quad \forall x \in B\left(x_{0}, \eta\right),
$$

with $\Psi_{x}(z):=x+c(x, z)$.

Assumption 2.4 implies the existence of a such a ball as we will explain now.

Let us fix $x$ and suppose first that $z \in \mathbb{R}$ and that $\Psi_{x}^{\prime}\left(z_{0}\right)=1$. Then a condition of the type $\left|\Psi_{x}^{\prime \prime}(z)\right| \leq K$, nearby $z_{0}$, will ensure that $\Psi_{x}$ is 'close' to the identity (plus a constant): there will exist consequently $B \subset \Psi_{x}\left(B\left(z_{0}, R\right)\right)$ 'large' enough for our future needs. In the multidimensional case, if $\nabla_{z} c\left(z_{0}, x\right)=\nabla_{z} \Psi_{x}\left(z_{0}\right)=\mathrm{Id}$, the same mechanism applies and (2.7) appears to be merely the multidimensional version of $\left|\Psi_{x}^{\prime \prime}(z)\right| \leq K$.

If $\nabla_{z} \Psi_{x}\left(z_{0}\right) \neq \mathrm{Id}$, we have to use an intermediate renormalized function

$$
f(z):=\left(\nabla_{z} c\left(z_{0}, x\right)\right)^{-1} \Psi_{x}(z)
$$

in order to use the preceding point, and the condition (2.7) leads again to the existence of a ball $\tilde{B} \subset f\left(B\left(z_{0}, R^{\prime}\right)\right)$. Now we use the condition (2.6) to transform $\tilde{B}$ into a set 'not flat' (i.e. containing the wanted ball $B$ ) included in $\Psi_{x}\left(B\left(z_{0}, R\right)\right)$.

This whole heuristic is basically the pattern behind the proof of Lemma 5.1 (stated and proved in Section 5); result which will help us to show the next proposition.

Proposition 2.6. Grant Assumptions 2.2,2.3 and 2.4. Fix $n_{0}$ such that $\left\{z \in \mathbb{R}^{d}:\left|z-z_{0}\right| \leq R\right\} \subset E_{n_{0}}$. Then there exist $\eta>0$ and some ball $B \subset \mathbb{R}^{d}$ such that for all $n \geq n_{0}$ and for all $V \in \mathcal{B}\left(\mathbb{R}^{d}\right)$, for all $k \geq 1$,

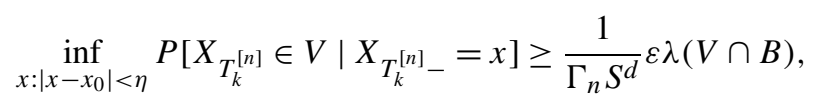

where $\lambda$ denotes the Lebesgue measure on $\mathbb{R}^{d}$. 
As a consequence of Proposition 2.6, the local Doeblin condition (2.4) holds with $C=\left\{x \in \mathbb{R}^{d}:\left|x-x_{0}\right|<\eta\right\}$,

$$
\beta=\frac{\lambda(B) \varepsilon}{\Gamma_{n} S^{d}} \wedge 1 \quad \text { and } \quad v(\mathrm{~d} y)=\frac{1}{\lambda(B)} 1_{B}(y) \mathrm{d} y .
$$

Notice that the set $C$ is not a 'petite' set in the sense of Meyn and Tweedie (1993) [11].

Remark 2.7. It is important to have a control on the radius $\eta$ of the set $C$, because return times to $C$ will serve as candidates for regeneration times. Such a control is possible if the jump height function $c(z, x)$ is Lipschitz continuous in $x$, uniformly in $z$, i.e. $L_{c}:=\sup _{z:\left|z-z_{0}\right| \leq R} L_{c}(z)<\infty$, where $L_{c}(z)$ has been introduced in Assumption 2.12 . In this case we can choose

$$
\eta=\frac{A\left(R \wedge \frac{1}{2 K}\right)}{4\left(1+L_{c}\right)} \wedge r
$$

see Lemma 5.1 in Section 5 below, and see the end of Section 5 for a proof of (2.11).

We close this section with two examples.

Example 2.8 (Growth-fragmentation processes). In reminiscence to Krell [6] (2015), we consider a one-dimensional growth-fragmentation-type process $X_{t}$ which is solution of

$$
\mathrm{d} X_{t}=\mathrm{d} t+\int_{\mathbb{R}_{+} \times \mathbb{R}_{+}}[\kappa(z)-1] \psi\left(X_{t-}\right) 1_{\left\{u \leq f\left(X_{t-}\right) / z\right\}} N(\mathrm{~d} t, \mathrm{~d} z, \mathrm{~d} u) .
$$

The ingredients of the above equation are

- the intensity measure $\mu(\mathrm{d} z)=1_{\mathbb{R}_{+}}(z) e^{-z} \mathrm{~d} z$;

- a 1-Lipschitz function $\psi: \mathbb{R}_{+} \rightarrow[a, b]$ which is a smooth version of $a \vee x \wedge b$ for $0<a<b<\infty$. The function $\psi$ is supposed to be increasing, such that $\psi(x) \equiv x$ for all $x \in[l, L]$ for some fixed thresholds $a<l<L<b$;

- an increasing, bounded Lipschitz function $f: \mathbb{R}_{+} \rightarrow \mathbb{R}_{+}$with $f(x)>0$ for all $x$;

- a function $\left.\left.\kappa: \mathbb{R}_{+} \rightarrow\right] \delta, 1\right], C^{2}$, Lipschitz continuous, with $\kappa(0)=1$ and $\kappa^{\prime}(z) \neq 0$ for all $z>0$.

Such a process serves e.g. as a model for the size of a marked Escherichia coli cell. The drift $g(x)=1$ implies that cells grow at deterministic rate 1 . Notice that there is no diffusive part here. At a jump time, a cell of size $l \leq x \leq L$ is replaced by a cell of size $\kappa(z) x$, and jumps happen at rate $\gamma(z, x)=f(x) \frac{1}{z}$ which is strictly positive for $z, x>0$.

It is straightforward to check that for this model, Assumptions 2.1-2.4 hold. The details are given at the end of Section 5 .

Example 2.9. We consider the one-dimensional case $d=1$ with $\mu(\mathrm{d} z)=\mathrm{d} z$. Throughout this example, $x_{0}$ and $r$ will be fixed and $\psi$ and $f$ will be bounded 1-Lipschitz functions such that $\psi(x)>0$ for all $x$ and $|f(x)| \geq \underline{f}>0$ for all $x$ such that $\left|x-x_{0}\right| \leq r$.

1. Suppose that $c(z, x)=\left[1-e^{-|z|}\right] f(x)$ and $\gamma(z, x)=\frac{1}{|z|} e^{-|z|} \psi(x)$. Then Assumption 2.1 holds, which can be seen as in Example 2.8 above. It is evident that Assumptions 2.2 and 2.3 hold. Finally, in order to check Assumption 2.4, fix $R$ and $z_{0}$ such that $\left|z_{0}\right| \geq R+a$, for some $a>0$. Then $\left|\frac{\partial c}{\partial z}\left(z_{0}, x\right)\right|=\left|f(x) e^{-\left|z_{0}\right|}\right| \geq A$ with $A=\underline{f} e^{-\left|z_{0}\right|}$, implying item 1. of Assumption 2.4. In order to check item 2. we notice that

$$
\left|\left(\frac{\partial c}{\partial z}\left(z_{0}, x\right)\right)^{-1}\right|\left|\frac{\partial^{2} c}{\partial z^{2}}(z, x)\right|=\frac{e^{\left|z_{0}\right|}}{|f(x)|}|f(x)| e^{-|z|} \leq e^{\left|z_{0}\right|-a}=: K,
$$

for all $z$ such that $\left|z-z_{0}\right| \leq R$. Item 3. clearly holds by continuity of $\frac{\partial c}{\partial z}(z, x)$ in $z$ and in $x$. 
2. We suppose now that $c(z, x)=f(x)\left[1+z^{2}\right]^{-1}$ and $\gamma(z, x)=\kappa(z) \psi(x)$, where $\kappa$ and $\psi$ are continuous functions taking values in some interval $[l, r]$, with $0<l<r<\infty$. Then Assumptions 2.1-2.3 hold. In order to check Assumption 2.4, fix $z_{0}$ and $R$ such that $\left|z_{0}\right| \geq R+a$, for some $a>0$. Then for all $x$ such that $\left|x-x_{0}\right| \leq r$,

$$
\left|\frac{\partial c}{\partial z}\left(z_{0}, x\right)\right|=\left|f(x) \frac{2 z_{0}}{\left(z_{0}^{2}+1\right)^{2}}\right| \geq A
$$

with $A=\frac{2 f\left|z_{0}\right|}{\left(z_{0}^{2}+1\right)^{2}}$. In the same way it can be seen that items 2. and 3. of Assumption 2.4 hold.

\subsection{Lyapunov condition}

The set $C=\left\{x \in \mathbb{R}^{d}:\left|x-x_{0}\right|<\eta\right\}$ appearing in the local Doeblin condition (2.4) and (2.9) will play the role of a small set in the sense of Nummelin [12] (1978) and Meyn-Tweedie [10] (1993). In order to be able to profit from the lower bound (2.9), we have to show that $\left(X_{T_{k}^{[n]}-}\right)_{k}$ comes back to the set $C$ infinitely often. For that sake, we introduce a Lyapunov condition in terms of the continuous time process, inspired by Douc, Fort and Guillin [3] (2009).

Assumption 2.10. There exists a continuous function $V: \mathbb{R}^{d} \rightarrow[1, \infty[$ which belongs to the domain $\mathcal{D}(L)$ of the extended generator $L$ of the process, an increasing concave positive function $\Phi:[1, \infty[\rightarrow(0, \infty)$ and a constant $b<\infty$ such that for any $x \in \mathbb{R}^{d}$,

$$
L V(x) \leq-\Phi \circ V(x)+b 1_{C^{\prime}}(x)
$$

where $C^{\prime}=\left\{x \in \mathbb{R}^{d}:\left|x-x_{0}\right|<\eta / 2\right\}, \eta$ as in Proposition 2.6.

This Lyapunov condition implies that $V\left(X_{t}\right)$ evolves as a nonnegative supermartingale as long as $X_{t}$ is in $\mathbb{R}^{d} \backslash C^{\prime}$. Hence a.s. the process $X_{t}$ with starting point $x \notin C^{\prime}$ will enter the set $C^{\prime}$ in finite time. This implies that the process comes back to the set $C^{\prime}=\left\{x:\left|x-x_{0}\right|<\eta / 2\right\}$ infinitely often. The choice of $\eta / 2$ is on purpose and will be explained by Proposition 3.3 below.

We discuss in Section 4 examples where (2.12) is verified.

Remark 2.11. Notice that since $\Phi$ is increasing strictly positive and since $V$ takes values in $[1, \infty[,(2.12)$ implies in particular that

$$
L V(x) \leq-c+b 1_{C^{\prime}}(x)
$$

for $c=\Phi(1)>0$. This is (2.12) for a constant function $\Phi \equiv c$. We will use (2.13) in order to show that $\left(X_{t}\right)_{t \geq 0}$ is positive Harris recurrent, in Section 3.3 below.

For the function $\Phi$ of (2.12) put

$$
H_{\Phi}(u)=\int_{1}^{u} \frac{\mathrm{d} s}{\Phi(s)}, \quad u \geq 1,
$$

and

$$
r_{\Phi}(s)=r(s)=\Phi \circ H_{\Phi}^{-1}(s) .
$$

Then Theorem 4.1 of Douc, Fort and Guillin [3] (2009) implies that for any $\delta>0$ and putting $\tau_{C^{\prime}}(\delta):=\inf \{t \geq \delta$ : $\left.X_{t} \in C^{\prime}\right\}$,

$$
E_{x} \int_{0}^{\tau_{C^{\prime}}(\delta)} r(s) \mathrm{d} s \leq V(x)+\frac{b}{\Phi(1)} \int_{0}^{\delta} r(s) \mathrm{d} s .
$$


In most of the cases, we will deal with the case $\Phi(v)=c v^{\alpha}$ for some fixed $0 \leq \alpha<1$ and $c>0$. In this case, it is easy to see that

$$
r(s)=c[c(1-\alpha) s+1]^{\frac{\alpha}{1-\alpha}}, \quad \text { and thus } \quad r(s) \sim C s^{\frac{\alpha}{1-\alpha}}, \quad \text { as } s \rightarrow \infty,
$$

where $C=c[c(1-\alpha)]^{\frac{\alpha}{1-\alpha}}$, giving rise to polynomial rates of convergence.

\subsection{Main results}

Theorem 2.12. Grant Assumptions 2.1, 2.2, 2.3, 2.4 and 2.10.

1. The process $X$ is recurrent in the sense of Harris having a unique invariant probability measure $m$ such that $\Phi \circ V \in \mathrm{L}^{1}(m)$. The invariant probability measure $m$ is the unique solution of

$$
\int_{\mathbb{R}^{d}} L \psi(x) m(\mathrm{~d} x)=0
$$

for all $\psi \in C^{2}\left(\mathbb{R}^{d}\right)$ being of compact support.

2. Moreover, for any measurable function $f \in \mathrm{L}^{1}(m)$, we have

$$
\frac{1}{t} \int_{0}^{t} f\left(X_{s}\right) \mathrm{d} s \rightarrow m(f)
$$

as $t \rightarrow \infty, P_{x}$-almost surely for any $x \in \mathbb{R}^{d}$.

The above ergodic theorem is an important tool e.g. for statistical inference based on observations of the process $X$ in continuous time. In this direction, the following deviation inequality is of particular interest. Recall that $v$ is the measure given in the local Doeblin condition (2.4).

Theorem 2.13. Grant the Assumptions 2.1, 2.2, 2.3, 2.4 and 2.10 with $\Phi(v)=c v^{\alpha}$, for some $0 \leq \alpha<1$ and $c \geq 1$. Put $p=1 /(1-\alpha)$. Let $f \in \mathrm{L}^{1}(m)$ with $\|f\|_{\infty}<\infty, x$ be any initial point and $0<\delta<\|f\|_{\infty}$. Then for all $t \geq 1$ the following inequality holds:

$$
\begin{aligned}
P_{x}\left(\left|\frac{1}{t} \int_{0}^{t} f\left(X_{S}\right) \mathrm{d} s-m(f)\right|>\delta\right) \leq & K(p, v, X) V(x) t^{-(p-1)} \\
& \times\left\{\begin{array}{ll}
\frac{1}{\delta^{2(p-1)}}\|f\|_{\infty}^{2(p-1)} & \text { if } p \geq 2, \\
\frac{1}{\delta^{p}}\|f\|_{\infty}^{p} & \text { if } 1<p<2
\end{array}\right\} .
\end{aligned}
$$

Here $K(p, v, X)$ is a positive constant, different in the two cases, which depends on $p, v$ and on the process $X$, but which does not depend on $f, t, \delta$.

Finally, we obtain the following quantitative control of the convergence of ergodic averages.

Proposition 2.14. Grant the Assumptions 2.1, 2.2, 2.3, 2.4 and 2.10 with $\Phi(v)=c v^{\alpha}$, for some $0 \leq \alpha<1$ and $c \geq 1$. Then for any $x, y \in \mathbb{R}^{d}$,

$$
\left\|\frac{1}{t} \int_{0}^{t} P_{s}(x, \cdot) \mathrm{d} s-\frac{1}{t} \int_{0}^{t} P_{s}(y, \cdot) \mathrm{d} s\right\|_{\mathrm{TV}} \leq C \frac{1}{t}\left(V(x)^{(1-\alpha)}+V(y)^{(1-\alpha)}\right),
$$

where $C>0$ is a constant. In particular, if $\alpha \geq \frac{1}{2}$, then

$$
\left\|\frac{1}{t} \int_{0}^{t} P_{S}(x, \cdot) \mathrm{d} s-m\right\|_{\mathrm{TV}} \leq C \frac{1}{t} V(x)^{(1-\alpha)} .
$$


We continue Example 2.8 and show that Theorem 2.12, Theorem 2.13 and Proposition 2.14 hold for growthfragmentation processes as considered there.

Example 2.15 (Example 2.8 continued). We continue the discussion of the growth-fragmentation model introduced in Example 2.8. We have already checked that Assumptions 2.1, 2.2, 2.3, 2.4 hold. It remains to check Assumption 2.10 with $\Phi(v)=c v^{\alpha}$, for some $0 \leq \alpha<1$. It is evident that in the dynamics of the growth-fragmentation process there is a competition between the deterministic growth rate of one cell, which is always 1, and pushes the process up, and the jumps, which make the process go down.

Recall that $\eta$ was given in (2.11). We introduce $V(x)=x^{2}+1$ and choose $x_{0}=0$. We will show at the end of Section 5 that under the following condition

$$
\int_{0}^{\infty} \frac{1}{z}(1-\kappa(z)) e^{-z} \mathrm{~d} z>\frac{2}{\psi(\eta / 2)}
$$

we have

$$
L V \leq-c(V-1)^{1 / 2}
$$

for all $x>\eta / 2$. Therefore, condition (2.13) holds with $\Phi(v)=c \sqrt{v-1}$, and as a consequence, Theorem 2.12, Theorem 2.13 and Proposition 2.14 hold.

Example 2.16. We continue Example 2.9 item 2. in the case when $x_{0}=0$ and $\sigma \equiv 0$. We suppose additionally that

$$
\langle x, g(x)\rangle \leq-c|x|^{1+\alpha}-D|x|
$$

for all $x \in \mathbb{R} \backslash C^{\prime}$, where $D:=\sup _{x \in \mathbb{R}}|f(x)| \psi(x) \int_{-\infty}^{\infty} \frac{1}{1+z^{2}} \kappa(z) \mathrm{d} z$. We show at the end of Section 4 below that in this case for $V(x)=|x|+1, L V(x) \leq-c|x|^{\alpha}$, for all $x \notin C^{\prime}$, and therefore, (2.13) holds with $\Phi(v)=c(v-1)^{\alpha}$, implying that Theorem 2.12, Theorem 2.13 and Proposition 2.14 hold.

The proofs of Theorems 2.12 and 2.13 and of Proposition 2.14 relies on the regeneration method that we are going to introduce now.

\section{Regeneration for the chain of big jumps}

\subsection{Regeneration times}

We show how the lower bound

$$
\Pi(x, \mathrm{~d} y) \geq 1_{C}(x) \beta \nu(\mathrm{d} y)
$$

of (2.4) for the jump kernel, with the choice of parameters in (2.10), allows us to introduce regeneration times for the process $X$.

We start 'splitting' the jump transition kernel $\Pi(x, \mathrm{~d} y)$ of $(2.3)$ in the following way. Since $\Pi(x, \mathrm{~d} y) \geq$ $\beta 1_{C}(x) v(\mathrm{~d} y)$, we may introduce a split kernel $Q((x, u), \mathrm{d} y)$, which is a transition kernel from $\mathbb{R}^{d} \times[0,1]$ to $\mathbb{R}^{\bar{d}}$, defined by

$$
Q((x, u), \mathrm{d} y)= \begin{cases}v(\mathrm{~d} y) & \text { if }(x, u) \in C \times[0, \beta], \\ \frac{1}{1-\beta}(\Pi(x, \mathrm{~d} y)-\beta v(\mathrm{~d} y)) & \text { if }(x, u) \in C \times] \beta, 1], \\ \Pi(x, \mathrm{~d} y) & \text { if } x \notin C .\end{cases}
$$

Notice that

$$
\int_{0}^{1} Q((x, u), \mathrm{d} y) \mathrm{d} u=\Pi(x, \mathrm{~d} y)
$$


it is in this sense that $Q((x, u), \mathrm{d} y)$ can be considered as 'splitting' the original transition kernel $\Pi$ by means of the additional 'color' $u$.

We now show how to construct a version of the process $X$ recursively over time intervals $\left[T_{k}^{[n]}, T_{k+1}^{[n]}[, k \geq 0\right.$. We start at time $t=0$ with $X_{0}=x$ and introduce the process $Z_{t}$ defined by

$$
Z_{t}=x+\int_{0}^{t} g\left(Z_{s}\right) \mathrm{d} s+\int_{0}^{t} \sigma\left(Z_{s}\right) \mathrm{d} W_{s}+\int_{0}^{t} \int_{E_{n}^{c}} \int_{0}^{\infty} c\left(z, Z_{s-}\right) 1_{u \leq \gamma\left(z, Z_{s-}\right)} N(\mathrm{~d} s, \mathrm{~d} z, \mathrm{~d} u)
$$

For $t<T_{1}^{[n]}$, we clearly have $Z_{t}=X_{t}$. Notice also that $T_{1}^{[n]}$ is independent of the r.h.s. of the above equation and exponentially distributed with parameter $\Gamma_{n}$. We put $X_{T_{1}^{[n]}-}:=Z_{T_{1}^{[n]}-}$ (notice that $Z_{T_{1}^{[n]}}=Z_{T_{1}^{[n]}-}$, since $Z$ almost surely does not jump at time $T_{1}^{[n]}$ ). On $X_{T_{1}^{[n]}-}=x^{\prime}$, we do the following.

1. We choose a uniform random variable $U_{1}$, uniformly distributed on $[0,1]$, independently of anything else.

2. On $U_{1}=u$, we choose a random variable $V_{1} \sim Q\left(\left(x^{\prime}, u\right), \mathrm{d} y\right)$ and we put

$$
X_{T_{1}^{[n]}}:=V_{1} .
$$

We then restart the above procedure with the new starting point $V_{1}$ instead of $x$.

We will write $\mathbf{X}_{t}$ for the $d+1$-dimensional process with additional color $U_{k}$, defined by

$$
\mathbf{X}_{t}=\sum_{k \geq 0} 1_{\left[T_{k}^{[n]}, T_{k+1}^{[n]}[\right.}(t)\left(X_{t}, U_{k}\right)
$$

i.e. $\mathbf{X}_{t}=\left(X_{t}, U_{k}\right)$ on each interval $\left[T_{k}^{[n]}, T_{k+1}^{[n]}\left[\right.\right.$, keeping trace of the additional color $U_{k}$.

Remark 3.1. Notice that the above splitting procedure does not even use the strong Markov property of the underlying process. It only uses the independence properties of the driving Poisson random measure.

This new process is clearly Markov with respect to its filtration, and by abuse of notation we will not distinguish between the original filtration $\mathbb{F}$ introduced in Section 2 and the canonical filtration of $\mathbf{X}_{t}$. In this richer structure, where we have added the component $U_{k}$ to the process, we obtain regeneration times for the process $\mathbf{X}$. More precisely, write

$$
A:=C \times[0, \beta]
$$

and put

$$
R_{0}:=0, \quad R_{k+1}:=\inf \left\{T_{m}^{[n]}>R_{k}: \mathbf{X}_{T_{m}^{[n]}-} \in A\right\} .
$$

Then we clearly have

\section{Proposition 3.2.}

(a) $\mathbf{X}_{R_{k}} \sim v(\mathrm{~d} x) 1_{[0,1]}(u)(\mathrm{d} u)$ on $R_{k}<\infty$, for all $k \geq 1$.

(b) $\mathbf{X}_{R_{k}+}$. is independent of $\mathcal{F}_{R_{k}}$ on $R_{k}<\infty$, for all $k \geq 1$.

(c) If $R_{k}<\infty$ for all $k$, then the sequence $\left(\mathbf{X}_{R_{k}}\right)_{k \geq 1}$ is i.i.d.

It is clear that in this way the speed of convergence to equilibrium of the process is determined by the moments of the extended stopping times $R_{k}$. In the next section we show that the drift condition of Assumption 2.10 ensures in particular that $R_{k}<\infty P_{x}$-almost surely for any $x$.

\subsection{Existence of moments of the regeneration times}

Recall the local Doeblin condition (2.4), the definition of the set $C=\left\{x:\left|x-x_{0}\right|<\eta\right\}$ and of $C^{\prime}=\left\{x:\left|x-x_{0}\right|<\right.$ $\eta / 2\}$. Let $\tau_{C^{\prime}}(\delta)=\inf \left\{t \geq \delta: X_{t} \in C^{\prime}\right\}$ be the first hitting time of $C^{\prime}$ after time $\delta$. Recall that condition (2.12) implies 
that

$$
E_{x} \int_{0}^{\tau}{ }^{\prime}(\delta) r(s) \mathrm{d} s \leq V(x)+\frac{b}{\Phi(1)} \int_{0}^{\delta} r(s) \mathrm{d} s,
$$

where $r$ is given as in (2.15), by Theorem 4.1 of Douc, Fort and Guillin [3] (2009).

In particular, equation (2.16) implies that $\tau_{C^{\prime}}<\infty P_{x}$-surely for all $x$. We show that this implies that the regeneration times $R_{k}$ introduced in (3.3) above are finite almost surely. Recall that $T_{k}^{[n]}$ are the successive jump times of the dominating Poisson point process $N^{[n]}$ having rate $\Gamma_{n}$. The regeneration times $R_{k}$ are expressed in terms of the jump chain $X_{T_{k}^{[n]}-}, k \geq 0$. We have to ensure that the control of return times to $C^{\prime}$ for the continuous time process imply analogous moments for the jump chain.

The main idea is to show that once the continuous time process has reached $C^{\prime}$, it takes some time to exit from $C$. Taking $\Gamma_{n}$, the rate of the dominating Poisson process, sufficiently large, the probability that a jump $T_{k}^{[n]}$ arises during this time, i.e. before exiting from $C$, can then be made arbitrarily large.

Proposition 3.3. There exists $n_{0}$, such that for any $n \geq n_{0}$,

$$
\inf _{x \in C^{\prime}} P_{x}\left(X_{T_{1}^{[n]}-} \in C\right) \geq \frac{1}{2} .
$$

Remark 3.4. The choice $\frac{1}{2}$ in the above lower bound is arbitrary, by choosing larger values of $n$, we could achieve any bound $1-\varepsilon$ on the right hand side of (3.5).

Proof. Before starting the proof, let $B$ be such that $|g(x)| \vee|\sigma(x)| \leq B(1+|x|), \forall x \in \mathbb{R}^{d}$. Since $g$ and $\sigma$ are supposed to be globally Lipschitz continuous, such a constant $B$ clearly exists.

Recall the process $Z_{t}$ defined by

$$
Z_{t}=x+\int_{0}^{t} g\left(Z_{s}\right) \mathrm{d} s+\int_{0}^{t} \sigma\left(Z_{s}\right) \mathrm{d} W_{s}+\int_{0}^{t} \int_{E_{n}^{c}} \int_{0}^{\infty} c\left(z, Z_{s-}\right) 1_{u \leq \gamma\left(z, Z_{s-}\right)} N(\mathrm{~d} s, \mathrm{~d} z, \mathrm{~d} u)
$$

and recall that for any $t<T_{1}^{[n]}, Z_{t}=X_{t}$. Recall also that $T_{1}^{[n]}$ is independent of the r.h.s. of the above equation, exponentially distributed with parameter $\Gamma_{n}$. Now let $x \in C^{\prime}$. We are looking for an upper-bound on

$$
\mathrm{P}_{x}\left[X_{T_{1}^{[n]}-} \notin C\right]=\mathrm{P}_{x}\left[Z_{T_{1}^{[n]}-} \notin C\right] \leq \mathrm{P}_{x}\left[\left|Z_{T_{1}^{[n]}-}-x\right|>\eta / 2\right] .
$$

Clearly, $\mathrm{P}_{x}\left[\left|Z_{t}-x\right| \geq \frac{\eta}{2}\right] \leq \frac{2}{\eta} E_{x}\left[\left|Z_{t}-x\right|\right]$, and we will therefore establish upper bounds on $E_{x}\left[\left|Z_{t}-x\right|\right]$. More precisely, we shall prove that there exist constants $d_{1}$ and $d_{2}$ such that

$$
\sup _{x \in C^{\prime}} E_{x}\left(\left|Z_{t}-x\right|\right) \leq d_{1} \sqrt{t} e^{d_{2} t} .
$$

In the sequel, we will be mainly interested in the small time behavior of the above control, i.e. the leading control in $t$ of the above formula is the term $\sqrt{t}-$ see also (3.11) below.

In order to prove (3.6), we proceed in two steps. In a first step, we obtain a control on $E_{x}\left(\sup _{t \leq T}\left|Z_{t}\right|\right)$. In a second step, we shall consider the deviation $E_{x}\left(\left|Z_{t}-x\right|\right)$.

Step 1. We first fix some $T>0$ and introduce $Z_{T}^{*}=\sup _{t \in[0, T]}\left|Z_{t}\right|$. Put moreover $\tau_{N}=\inf \left\{t \geq 0: Z_{t}^{*} \geq N\right\}$. Then for any fixed $N \geq 1$,

$$
\begin{array}{r}
E_{x}\left[Z_{T \wedge \tau_{N}}^{*}\right] \leq|x|+E_{x}\left[\sup _{t \leq T}\left|\int_{0}^{t} \sigma\left(Z_{s \wedge \tau_{N}}\right) \mathrm{d} W_{s}\right|\right]+E_{x}\left[\int_{0}^{T}\left|g\left(Z_{s \wedge \tau_{N}}\right)\right| \mathrm{d} s\right] \\
+E_{x}\left[\int_{0}^{T \wedge \tau_{N}} \int_{E_{n}^{c}} \int_{0}^{\infty}\left|c\left(z, Z_{s-}\right)\right| 1_{u \leq \gamma\left(z, Z_{s-}\right)} N(\mathrm{~d} s, \mathrm{~d} z, \mathrm{~d} u)\right]
\end{array}
$$




$$
\begin{array}{r}
\leq|x|+E_{x}\left[\sup _{t \leq T}\left|\int_{0}^{t} \sigma\left(Z_{s \wedge \tau_{N}}\right) \mathrm{d} W_{s}\right|\right]+E_{x}\left[\int_{0}^{T}\left|g\left(Z_{s \wedge \tau_{N}}\right)\right| \mathrm{d} s\right] \\
+E_{x}\left[\int_{0}^{T \wedge \tau_{N}} \int_{E_{n}^{c}} \int_{0}^{\infty}\left|c\left(z, Z_{s-}\right)\right| 1_{u \leq \gamma\left(z, Z_{s-}\right)} N(\mathrm{~d} s, \mathrm{~d} z, \mathrm{~d} u)\right] .
\end{array}
$$

We follow the arguments of the proof of Theorem 1.2 of Graham [5] (1992) and use the Burkholder-Davis-Gundy inequality in order to get

$$
E_{x}\left[\sup _{t \leq T}\left|\int_{0}^{t} \sigma\left(Z_{s \wedge \tau_{N}}\right) \mathrm{d} W_{s}\right|\right] \leq C(1) E_{x}\left[\left(\left|\int_{0}^{T} \sigma^{2}\left(Z_{s \wedge \tau_{N}}\right) \mathrm{d} s\right|\right)^{1 / 2}\right] .
$$

But

$$
\left|\sigma\left(Z_{S \wedge \tau_{N}}\right)\right| \leq B\left(1+\left|Z_{S \wedge \tau_{N}}\right|\right) \leq B\left(1+Z_{T \wedge \tau_{N}}^{*}\right) .
$$

This implies that

$$
E_{x}\left[\sup _{t \leq T}\left|\int_{0}^{t} \sigma\left(Z_{s \wedge \tau_{N}}\right) \mathrm{d} W_{s}\right|\right] \leq C(1) B \sqrt{T}\left[1+E_{x} Z_{T \wedge \tau_{N}}^{*}\right] .
$$

The same argument shows that

$$
\begin{aligned}
E_{x}\left[\int_{0}^{T}\left|g\left(Z_{s \wedge \tau_{N}}\right)\right| \mathrm{d} s\right] & \leq E_{x}\left[\int_{0}^{T} B\left(1+\left|Z_{s \wedge \tau_{N}}\right|\right) \mathrm{d} s\right] \\
& \leq B T\left[1+E_{x} Z_{T \wedge \tau_{N}}^{*}\right] .
\end{aligned}
$$

Finally, we upper bound

$$
\begin{aligned}
& E_{x}\left[\int_{0}^{T \wedge \tau_{N}} \int_{E_{n}^{c}} \int_{0}^{\infty}\left|c\left(z, Z_{s-}\right)\right| 1_{u \leq \gamma\left(z, Z_{s-}\right)} N(\mathrm{~d} s, \mathrm{~d} z, \mathrm{~d} u)\right] \\
& =E_{x}\left[\int_{0}^{T} \int_{E_{n}^{c}} \int_{0}^{\infty}\left|c\left(z, Z_{s-}\right)\right| 1_{u \leq \gamma\left(z, Z_{s-}\right)} \mathrm{d} z \mathrm{~d} \mu(z) \mathrm{d} u\right] \\
& \leq T \sup _{x} \int_{E_{n}^{c}}|c(z, x)| \gamma(z, x) \mathrm{d} \mu(z) \leq T \sup _{x} \int_{\mathbb{R}^{d}}|c(z, x)| \gamma(z, x) \mathrm{d} \mu(z)
\end{aligned}
$$

and put

$$
D_{1}=\sup _{x} \int_{\mathbb{R}^{d}}|c(z, x)| \gamma(z, x) \mathrm{d} \mu(z)+[C(1)+1] B, \quad D_{2}=[C(1)+1] B .
$$

Now, fix some parameter $0<a<1$ and choose $T<1$ sufficiently small such that $D_{2} \sqrt{T} \leq a$ and such that moreover $D_{1} \sqrt{T}<1$. Notice that since $T<1$, we have $\sqrt{T} \geq T$.

Therefore, resuming the above steps,

$$
E_{x}\left[Z_{T \wedge \tau_{N}}^{*}\right] \leq|x|+D_{1} \sqrt{T}+D_{2} \sqrt{T} E_{x}\left(Z_{T \wedge \tau_{N}}^{*}\right) \leq|x|+1+a E_{x}\left(Z_{T \wedge \tau_{N}}^{*}\right)
$$

Since $a<1$, we obtain

$$
E_{x}\left[Z_{T \wedge \tau_{N}}^{*}\right] \leq \frac{1}{1-a}(|x|+1)
$$


and letting $N \rightarrow \infty$ implies the same upper bound

$$
E_{x}\left[Z_{T}^{*}\right] \leq \frac{1}{1-a}(|x|+1) .
$$

Iterating the above procedure and using the Markov property, we obtain

$$
E_{x}\left[\sup _{(n-1) T \leq t \leq n T}\left|Z_{t}\right|\right] \leq\left(\sum_{k=1}^{n} \frac{1}{(1-a)^{k}}\right) 1+\frac{1}{(1-a)^{n}}|x| \leq \frac{1}{a} e^{\log \left(\frac{1}{1-a}\right) n}[1+|x|] .
$$

Now fix $t>0$ and let $n$ be such that $(n-1) T \leq t \leq n T$. Hence $n \leq t / T+1$ and

$$
E_{x}\left[\left|Z_{t}\right|\right] \leq E_{x}\left[\sup _{(n-1) T \leq t \leq n T}\left|Z_{t}\right|\right] \leq \frac{1}{a} e^{\log \left(\frac{1}{1-a}\right)\left(\frac{t}{T}+1\right)}[1+|x|] .
$$

Observing that $|x| \leq\left|x_{0}\right|+\eta / 2$ for $x \in C^{\prime}$, it follows from (3.9) that for $c_{1}=c_{1}\left(x_{0}, \eta\right)=\frac{1}{a} e^{\log \left(\frac{1}{1-a}\right)}\left(1+\left|x_{0}\right|+\eta / 2\right)$ and $c_{2}=\log \left(\frac{1}{1-a}\right) \cdot \frac{1}{T}$,

$$
\sup _{x \in C^{\prime}} E_{x}\left[\left|Z_{t}\right|\right] \leq c_{1} e^{c_{2} t},
$$

for all $t \geq 0$ (let us emphasize that we are interested here in the case where $t$ is 'small,' so the exponential behavior of this upper bound is not a concern in our framework).

Step 2. In order to prove (3.6), we now iterate the arguments that led to (3.8) and obtain in the same way

$$
E_{x}\left[\sup _{t \leq T}\left|Z_{t}-x\right|\right] \leq \frac{1}{1-a} D_{1} \sqrt{T}(1+|x|),
$$

but now we keep the factor $\sqrt{T}$ explicitly since it will be important later.

Using a telescopic sum and the Markov property gives

$$
E_{x}\left[\sup _{(n-1) T \leq t \leq n T}\left|Z_{t}-x\right|\right] \leq \sum_{k=1}^{n} E_{x}\left[\sup _{(k-1) T \leq t \leq k T}\left|Z_{t}-Z_{(k-1) T}\right|\right] \leq \frac{D_{1} \sqrt{T}}{1-a} \sum_{k=1}^{n} E_{x}\left[1+\left|Z_{(k-1) T}\right|\right] .
$$

By (3.10), for all $k \leq n, E_{x}\left[1+\left|Z_{(k-1) T}\right|\right] \leq 1+c_{1} e^{c_{2} n T} \leq\left[1+c_{1}\right] e^{c_{2} n T}$, if $x \in C^{\prime}$. Hence

$$
\sup _{x \in C^{\prime}} E_{x}\left[\sup _{(n-1) T \leq t \leq n T}\left|Z_{t}-x\right|\right] \leq \frac{D_{1} \sqrt{T}}{1-a} n\left[1+c_{1}\right] e^{c_{2} n T} .
$$

Using that $n e^{c_{2} n T} \leq c_{3} e^{c_{3} n T}$ for some constant $c_{3}$, and using the same trick that allowed to deduce (3.10) from (3.9), this implies (3.6), for suitable constants $d_{1}$ and $d_{2}$.

Now, we choose $n$ such that $\Gamma_{n}>d_{2}$. Recall that $T_{1}^{[n]}$ is independent from $\left(Z_{t}\right)_{t}$, exponentially distributed with parameter $\Gamma_{n}$. Moreover, almost surely, $Z$ does not jump at $T_{1}^{[n]}$. Therefore,

$$
\begin{aligned}
E_{x}\left[\left|Z_{T_{1}^{[n]}-}-x\right|\right] & =E_{x}\left[\left|Z_{T_{1}^{[n]}}-x\right|\right] \leq \int_{0}^{+\infty} d_{1} \sqrt{t} e^{d_{2} t} \Gamma_{n} e^{-\Gamma_{n} t} \mathrm{~d} t \\
& =\Gamma_{n} d_{1} \int_{0}^{+\infty} \sqrt{t} e^{-\left(\Gamma_{n}-d_{2}\right) t} \mathrm{~d} t=\Gamma_{n} d_{1} \frac{\Gamma\left(\frac{3}{2}\right)}{\left(\Gamma_{n}-d_{2}\right)^{\frac{3}{2}}}=\Gamma_{n} d_{1} \frac{\sqrt{\pi}}{2} \frac{1}{\left(\Gamma_{n}-d_{2}\right)^{\frac{3}{2}}}
\end{aligned}
$$

for every $x \in C^{\prime}$. We choose $n_{0}$ such that for all $n \geq n_{0}$,

$$
\Gamma_{n} d_{1} \frac{\sqrt{\pi}}{2} \frac{1}{\left(\Gamma_{n}-d_{2}\right)^{\frac{3}{2}}}<\frac{\eta}{4}
$$


and obtain

$$
\begin{aligned}
\sup _{x \in C^{\prime}} \mathrm{P}_{x}\left[X_{T_{1}^{[n]}-} \notin C\right] & \leq \sup _{x \in C^{\prime}} \mathrm{P}_{x}\left[\left|Z_{T_{1}^{[n]}-}-x\right| \geq \frac{\eta}{2}\right] \\
& \leq \sup _{x \in C^{\prime}} \frac{2}{\eta} E_{x}\left[\left|Z_{T_{1}^{[n]}-}-x\right|\right]<\frac{1}{2} .
\end{aligned}
$$

Let $S_{1}=\inf \left\{T_{k}^{[n]}, k \geq 1: X_{T_{k}^{[n]}-} \in C\right\}$. Then the above arguments imply the following statement.

Corollary 3.5. $P_{x}\left(S_{1}<\infty\right)=1$ for all $x$.

Proof. We introduce the following sequence of stopping times.

$$
\begin{aligned}
& t_{1}=\tau_{C^{\prime}}, \quad s_{1}=\inf \left\{T_{k}^{[n]}: T_{k}^{[n]}>t_{1}\right\}, \quad \ldots, \\
& t_{l}=\inf \left\{s \geq s_{l-1}: X_{s} \in C^{\prime}\right\}, \quad s_{l}=\inf \left\{T_{k}^{[n]}: T_{k}^{[n]}>t_{l}\right\} .
\end{aligned}
$$

The above stopping times are all finite almost surely. We put

$$
\tau_{*}=\inf \left\{l: X_{s_{l}-} \in C\right\} .
$$

Then, using (3.5), for any $x \in \mathbb{R}^{d}$,

$$
P_{x}\left(\tau_{*} \geq n_{0}\right) \leq\left(\frac{1}{2}\right)^{n_{0}}
$$

which shows that $\tau_{*}<\infty P_{x}$-almost surely for all $x$. In particular,

$$
S_{1} \leq s_{\tau_{*}}<\infty
$$

$P_{x}$-almost surely for all $x$.

In case that $\Phi(v)=c v^{\alpha}$ for some fixed $0 \leq \alpha<1$, by (2.17) we know that $r(s) \leq C s^{\alpha /(1-\alpha)}$, and in this case (2.16) provides a polynomial control obtained for the first entrance time in $C^{\prime}$. We now show that this polynomial control remains true for $S_{1}$.

Proposition 3.6. Grant Assumption 2.10 with $\Phi(v)=c v^{\alpha}$, for some $0 \leq \alpha<1$ and $c \geq 1$. Then there exists a constant C such that

$$
E_{x}\left(S_{1}^{\frac{1}{1-\alpha}}\right) \leq C V(x)
$$

Proof. We adopt the notations of the proof of Corollary 3.5 and start with some preliminary considerations concerning the rate function introduced in (2.17) above.

0 . For rate functions as in (2.17), the most important technical feature is the following sub-additivity property

$$
r(t+s) \leq C(r(t)+r(s)),
$$

for $t, s \geq 0$ and $C$ a positive constant. We shall also use that for (2.17),

$$
r(t+s) \leq r(t) r(s),
$$

for all $t, s \geq 0$, which follows from the sub-linearity of $\mathbb{R}_{+} \ni x \mapsto \ln (1+x)$ and the fact that the constant $c$ in the formula of $r(s)$ in (2.17) is strictly larger than $1 .^{1}$

\footnotetext{
${ }^{1}$ Supposing that $c \geq 1$ is actually no restriction, since we might multiply (2.12) by any suitably large constant.
} 
1. Since $r(s) \geq c[c(1-\alpha)]^{\alpha /(1-\alpha)} s^{\alpha /(1-\alpha)}$ by (2.17), it is sufficient to show that

$$
E_{x} \int_{0}^{s_{\tau_{*}}} r(s) \mathrm{d} s \leq \tilde{C} V(x),
$$

for a suitable constant $\tilde{C}$, in order to prove (3.13). Indeed, (3.16) and the fact that $S_{1} \leq s_{\tau_{*}}$ imply that

$$
E_{x} \int_{0}^{S_{1}} s^{\alpha /(1-\alpha)} \mathrm{d} s \leq \tilde{C} V(x) .
$$

Then (3.13) follows, since $\int_{0}^{S_{1}} s^{\alpha /(1-\alpha)} \mathrm{d} s=C S_{1}^{\frac{1}{1-\alpha}}$.

We are going to prove (3.16) in the next steps. In what follows, $C$ will denote a constant that might change from line to line and even within the same line.

2. We start by studying $E_{x} \int_{0}^{s_{1}} r(s) \mathrm{d} s$, where $s_{1}=\inf \left\{T_{k}^{[n]}: T_{k}^{[n]}>\tau_{C^{\prime}}\right\}$. Recall that $\Gamma_{n}$ is the rate of the Poisson process associated to $T_{k}^{[n]}, k \geq 1$. Then by definition of $s_{1}$,

$$
E_{x} \int_{0}^{s_{1}} r(s) \mathrm{d} s=E_{x} \int_{0}^{\tau^{\tau^{\prime}}} r(s) \mathrm{d} s+E_{x} \int_{\tau_{C^{\prime}}}^{s_{1}} r(s) \mathrm{d} s \leq V(x)+E_{x} \int_{\tau_{C^{\prime}}}^{s_{1}} r(s) \mathrm{d} s,
$$

where we have used (2.16) with $\delta=0$.

Now, using that $s_{1}-\tau_{C^{\prime}}$ is independent of $\mathcal{F}_{\tau_{C^{\prime}}}$, exponentially distributed with parameter $\Gamma_{n}$, and using (3.15),

$$
\begin{aligned}
E_{x} \int_{\tau_{C^{\prime}}}^{s_{1}} r(s) \mathrm{d} s & =E_{x} E_{X_{\tau C^{\prime}}} \int_{0}^{s_{1}-\tau_{C^{\prime}}} r\left(\tau_{C^{\prime}}+s\right) \mathrm{d} s \\
& \leq E_{x} E_{X_{\tau C^{\prime}}} \int_{0}^{s_{1}-\tau_{C^{\prime}}} r\left(\tau_{C^{\prime}}\right) r(s) \mathrm{d} s \\
& =E_{x}\left(r\left(\tau_{C^{\prime}}\right)\right) E_{x} E_{X_{\tau_{C^{\prime}}}}\left(\int_{0}^{s_{1}-\tau_{C^{\prime}}} r(s) \mathrm{d} s\right) \\
& =E_{x}\left(r\left(\tau_{C^{\prime}}\right)\right) E_{x} E_{X_{\tau_{C^{\prime}}}}\left(\int_{0}^{\infty} \Gamma_{n} e^{-\Gamma_{n} t}\left[\int_{0}^{t} r(s) \mathrm{d} s\right] \mathrm{d} t\right) \\
& =C_{1} E_{x}\left(r\left(\tau_{C^{\prime}}\right)\right),
\end{aligned}
$$

where $C_{1}=\int_{0}^{\infty} \Gamma_{n} e^{-\Gamma_{n} t}\left[\int_{0}^{t} r(s) \mathrm{d} s\right] \mathrm{d} t<\infty$.

Notice that $\lim _{t \rightarrow \infty} \frac{r(t)}{\int_{0}^{t} r(s) \mathrm{d} s}=0$, by (2.17). This implies that there exists a suitable constant $C$ such that

$$
r(t) \leq C+\int_{0}^{t} r(s) \mathrm{d} s, \quad \text { for all } t \geq 0 .
$$

Therefore,

$$
E_{x}\left(r\left(\tau_{C^{\prime}}\right)\right) \leq C+E_{x} \int_{0}^{\tau_{C^{\prime}}} r(s) \mathrm{d} s \leq C+V(x)
$$

by (2.16) with $\delta=0$, implying that

$$
E_{x} \int_{0}^{s_{1}} r(s) \mathrm{d} s \leq C_{1} \cdot C+C_{1} V(x) \leq\left[C_{1} C+C_{1}\right] V(x),
$$

where we have used that $V(x) \geq 1$. 
3. In a next step we consider the following situation. Let $x \in C^{\prime}$, then $t_{1}=0$ and $t_{2}=\inf \left\{t \geq s_{1}: X_{t} \in C^{\prime}\right\}$. Since $s_{1}$ is independent of the process, exponentially distributed with parameter $\Gamma_{n}$, we may apply (2.16) in order to obtain, for $x \in C^{\prime}$,

$$
\begin{aligned}
E_{x} \int_{0}^{t_{2}} r(s) \mathrm{d} s & =\int_{0}^{\infty} \Gamma_{n} e^{-\Gamma_{n} t}\left(E_{x} \int_{0}^{\tau_{C^{\prime}}(t)} r(s) \mathrm{d} s\right) \mathrm{d} t \\
& \leq \int_{0}^{\infty} \Gamma_{n} e^{-\Gamma_{n} t}\left(V(x)+\frac{b}{c} \int_{0}^{t} r(s) \mathrm{d} s\right) \mathrm{d} t \\
& \leq V(x)+C\left(\Gamma_{n} b, c\right),
\end{aligned}
$$

where $C\left(\Gamma_{n} b, c\right)$ is a constant depending on $\Gamma_{n}, b$ and $c=\Phi(1)$, since $t \mapsto \int_{0}^{t} r(s) \mathrm{d} s$ is of polynomial growth and therefore integrable with respect to $\Gamma_{n} e^{-\Gamma_{n} t} \mathrm{~d} t$. As a consequence,

$$
\sup _{x \in C^{\prime}} E_{x} \int_{0}^{t_{2}} r(s) \mathrm{d} s<\infty .
$$

4. We now use $r(t+s) \leq r(t) r(s)$ in order to obtain a control of $E_{x} \int_{0}^{t_{\tau *}} r(s) \mathrm{d} s$. We certainly have

$$
\begin{aligned}
E_{x} \int_{0}^{t_{\tau_{*}}} r(s) \mathrm{d} s & =E_{x} \int_{0}^{t_{1}} r(s) \mathrm{d} s+\sum_{n \geq 1} E_{x} \int_{t_{n}}^{t_{n+1}} r(s) \mathrm{d} s 1_{\left\{n<\tau_{*}\right\}} \\
& \leq V(x)+\sum_{n \geq 1} E_{x}\left(1_{\left\{n-1<\tau_{*}\right\}} \int_{0}^{t_{n+1}-t_{n}} r\left(t_{n}+s\right) \mathrm{d} s\right) .
\end{aligned}
$$

Since $r\left(t_{n}+s\right) \leq r\left(t_{n}\right) r(s)$, we may continue as follows.

$$
\begin{aligned}
E_{x} \int_{0}^{t_{\tau_{*}}} r(s) \mathrm{d} s & \leq V(x)+\sum_{n \geq 1} E_{x}\left(1_{\left\{n-1<\tau_{*}\right\}} r\left(t_{n}\right) \int_{0}^{t_{n+1}-t_{n}} r(s) \mathrm{d} s\right) \\
& =V(x)+\sum_{n \geq 1} E_{x}\left(1_{\left\{n-1<\tau_{*}\right\}} r\left(t_{n}\right) E_{X_{t_{n}}} \int_{0}^{t_{1}} r(s) \mathrm{d} s\right) \\
& \leq V(x)+\sum_{n \geq 1} E_{x}\left(1_{\left\{n-1<\tau_{*}\right\}} r\left(t_{n}\right) V\left(X_{t_{n}}\right)\right),
\end{aligned}
$$

where we have used (2.16) and the fact that $1_{\left\{n-1<\tau_{*}\right\}}$ is $\mathcal{F}_{s_{n-1}}$-measurable. Now, $X_{t_{n}}$ belonging to $C^{\prime}$, we can upperbound $V\left(X_{t_{n}}\right) \leq \sup _{x \in C^{\prime}} V(x)=:\|V\|_{\infty, C^{\prime}}$, and obtain

$$
E_{x} \int_{0}^{t_{\tau_{*}}} r(s) \mathrm{d} s \leq V(x)+\|V\|_{\infty, C^{\prime}} \sum_{n \geq 1} E_{x}\left(1_{\left\{n-1<\tau_{*}\right\}} r\left(t_{n}\right)\right) .
$$

We use that by definition of the stopping times $t_{n}, t_{n}=t_{1}+t_{n-1} \circ \theta_{t_{1}}$, where $\theta_{t_{1}}$ denotes the shift operator on $D\left(\mathbb{R}_{+}, \mathbb{R}^{d}\right)$. But

$$
r\left(t_{n}\right)=r\left(t_{1}+t_{n-1} \circ \theta_{t_{1}}\right) \leq r\left(t_{1}\right) r\left(t_{n-1} \circ \theta_{t_{1}}\right),
$$

due to (3.15). Using the Markov property with respect to $t_{1}$ and the fact that $X_{t_{1}} \in C^{\prime}$, we obtain

$$
E_{x}\left(1_{\left\{n-1<\tau_{*}\right\}} r\left(t_{n}\right)\right) \leq E_{x} r\left(t_{1}\right) \sup _{y \in C^{\prime}} E_{y}\left(r\left(t_{n-1}\right) 1_{\left\{n-2<\tau_{*}\right\}}\right) .
$$


Using (3.17), the first factor can be treated as follows

$$
E_{x} r\left(t_{1}\right) \leq C+E_{x} \int_{0}^{t_{1}} r(s) \mathrm{d} s \leq C+V(x) \leq[C+1] V(x),
$$

since $V(x) \geq 1$.

We are now going to treat the second factor. For that sake, let $p \in] \frac{1-\alpha}{\alpha} \vee 1, \frac{1}{\alpha}\left[\right.$, and $q \geq 1$ with $\frac{1}{p}+\frac{1}{q}=1$. Then, using that $P\left(n-2<\tau_{*}\right) \leq\left(\frac{1}{2}\right)^{n-2}$,

$$
E_{y}\left(1_{\left\{n-2<\tau_{*}\right\}} r\left(t_{n-1}\right)\right) \leq E_{y}\left(r^{p}\left(t_{n-1}\right)\right)^{1 / p}\left(\frac{1}{2}\right)^{(n-2) / q} .
$$

We use the precise form of $r$ given in (2.17) and obtain, using Hölder's inequality, the fact that $\frac{\alpha p}{1-\alpha}>1$ and writing that $t_{n-1}=t_{1}+\left(t_{2}-t_{1}\right)+\cdots+\left(t_{n-1}-t_{n-2}\right)$,

$$
r^{p}\left(t_{n-1}\right) \leq(n-1)^{\frac{\alpha p}{1-\alpha}-1}\left[r^{p}\left(t_{1}\right)+\cdots+r^{p}\left(t_{n-1}-t_{n-2}\right)\right] .
$$

Our intention is now to show that for all $t, r^{p}(t) \leq C \int_{0}^{t} r(s) \mathrm{d} s$, for a suitable constant $C$. In order to do so, recall that by (2.17), $r^{p}(t) \leq C t^{\frac{\alpha}{1-\alpha} p}$, as $t \rightarrow \infty$. Since $p<1 / \alpha$, we have $\frac{\alpha}{1-\alpha} p-1 \leq \frac{\alpha}{1-\alpha} \frac{1}{\alpha}-1=\frac{\alpha}{1-\alpha}$. Moreover, $\frac{\alpha}{1-\alpha} p>1$, since $p>\frac{1-\alpha}{\alpha}$. Therefore,

$$
t^{\frac{\alpha}{1-\alpha}} p=\frac{1}{\frac{\alpha}{1-\alpha} p-1} \int_{0}^{t} s^{\frac{\alpha}{1-\alpha} p-1} \mathrm{~d} s=\frac{1}{\frac{\alpha}{1-\alpha} p-1} \int_{0}^{t} s^{\frac{\alpha}{1-\alpha}} \mathrm{d} s \leq C \int_{0}^{t} r(s) \mathrm{d} s,
$$

implying the desired assertion.

As a consequence we may upper bound each term appearing in (3.27) by $r^{p}\left(t_{1}\right) \leq C \int_{0}^{t_{1}} r(s) \mathrm{d} s, \ldots, r^{p}\left(t_{n-1}-\right.$ $\left.t_{n-2}\right) \leq C \int_{0}^{t_{n-1}-t_{n-2}} r(s) \mathrm{d} s$ and obtain, for a new constant $C$,

$$
r^{p}\left(t_{n-1}\right) \leq C(n-1)^{\frac{\alpha p}{1-\alpha}-1}\left(\int_{0}^{t_{1}} r(s) \mathrm{d} s+\cdots+\int_{0}^{t_{n-1}-t_{n-2}} r(s) \mathrm{d} s\right) .
$$

Using successively the Markov property at times $0, t_{1}, t_{2}, \ldots, t_{n-2}$ and that $X_{t_{i}} \in C^{\prime}$, for $1 \leq i \leq n-2$, we obtain

$$
E_{y} r^{p}\left(t_{n-1}\right) \leq C(n-1)^{\frac{\alpha p}{1-\alpha}} \sup _{z \in C^{\prime}} E_{z} \int_{0}^{t_{2}} r(s) \mathrm{d} s .
$$

Finally, by (3.20), $\sup _{z \in C^{\prime}} E_{z} \int_{0}^{t_{2}} r(s) \mathrm{d} s<\infty$, and therefore

$$
\sup _{y \in C^{\prime}}\left(E_{y} r^{p}\left(t_{n-1}\right)\right)^{1 / p} \leq C(n-1)^{\frac{\alpha}{1-\alpha}} .
$$

Coming back to (3.23) and using (3.24)-(3.26), we conclude that, for a suitable constant $C$,

$$
E_{x} \int_{0}^{t_{\tau^{*}}} r(s) \mathrm{d} s \leq V(x)+C V(x) \sum_{n \geq 1}\left(\frac{1}{2}\right)^{\frac{n-2}{q}}(n-1)^{\frac{\alpha}{1-\alpha}} \leq \tilde{C} V(x) .
$$

5. We conclude as follows. For a constant $C$ that might change from line to line,

$$
\begin{aligned}
E_{x} \int_{0}^{s_{\tau^{*}}} r(s) \mathrm{d} s & =E_{x} \int_{0}^{t_{\tau^{*}}} r(s) \mathrm{d} s+E_{x} \int_{t_{\tau^{*}}}^{s_{\tau^{*}}} r(s) \mathrm{d} s \\
& \leq C V(x)+\sum_{n \geq 1} E_{x} 1_{\left\{\tau^{*}=n\right\}} \int_{t_{n}}^{s_{n}} r(s) \mathrm{d} s
\end{aligned}
$$




$$
\begin{aligned}
& \leq C V(x)+\sum_{n \geq 1} E_{x} 1_{\left\{\tau^{*}>n-1\right\}} r\left(t_{n}\right) \int_{0}^{s_{n}-t_{n}} r(s) \mathrm{d} s \\
& \leq C V(x)+\sum_{n \geq 1} E_{x}\left[1_{\left\{\tau^{*}>n-1\right\}} r\left(t_{n}\right) \sup _{y \in C^{\prime}} E_{y} \int_{0}^{s_{1}} r(s) \mathrm{d} s\right] \\
& \leq C V(x)+\left(\sup _{y \in C^{\prime}} E_{y} \int_{0}^{s_{1}} r(s) \mathrm{d} s\right) \sum_{n \geq 1} E_{x}\left[1_{\left\{\tau^{*}>n-1\right\}} r\left(t_{n}\right)\right] \\
& \leq C V(x)+C \sum_{n \geq 1} E_{x} 1_{\left\{\tau^{*}>n-1\right\}} r\left(t_{n}\right),
\end{aligned}
$$

where we have used that $r\left(t_{n}+s\right) \leq r\left(t_{n}\right) r(s)$, the Markov property with respect to $t_{n}$ and the fact that by (3.18),

$$
\sup _{y \in C^{\prime}} E_{y} \int_{0}^{s_{1}} r(s) \mathrm{d} s<\infty .
$$

The last sum $\sum_{n \geq 1} E_{x} 1_{\left\{\tau^{*}>n-1\right\}} r\left(t_{n}\right)$ is treated as (3.23), and this concludes our proof.

The above result implies an analogous control for moments of the regeneration times $R_{k}$ of (3.3). More precisely, we can now define

$$
S_{l}=\inf \left\{T_{k}^{[n]}>S_{l-1}: X_{T_{k}^{[n]}-} \in C\right\}, \quad l \geq 2,
$$

and let

$$
R_{1}=\inf \left\{S_{l}: U_{l} \leq \beta\right\}, \quad R_{k+1}=\inf \left\{S_{l}>R_{k}: U_{l} \leq \beta\right\} .
$$

An analogous argument as the one used in the proof of Proposition 3.6 then implies the following theorem.

Theorem 3.7. Grant Assumption 2.10 with $\Phi(v)=c v^{\alpha}$, for some $0 \leq \alpha<1$, and let $p=1 /(1-\alpha)$. Then

$$
E_{x} R_{1}^{p} \leq C V(x)
$$

We are now ready to prove Theorems 2.12 and 2.13 .

\subsection{Proofs of Theorems 2.12 and 2.13}

Proof of Theorem 2.12. Let

$$
\mathbf{m}(O):=E \int_{R_{1}}^{R_{2}} 1_{O}\left(\mathbf{X}_{s}\right) \mathrm{d} s,
$$

for any measurable set $O$. By the strong law of large numbers, any set $O$ with $\mathbf{m}(O)>0$ is visited i.o. $P_{x}$-almost surely by the process $\mathbf{X}$, for any starting point $(x, u) \in \mathbb{R}^{d} \times[0,1]$. Hence, the process is recurrent in the sense of Harris, and by the Kac occupation time formula, $\mathbf{m}$ is the unique invariant measure of the process (unique up to multiplication with a constant).

Now, recall that $v$ is of compact support, hence $V \in L^{1}(v)$. Recall that by Remark 2.11, the Lyapunov condition (2.12) holds in particular for a constant function $\Phi \equiv \Phi(1)=c$, i.e. $\Phi(v)=c v^{\alpha}$ with $\alpha=0$. Using (3.29) in the case $\alpha=0$, we obtain $\mathbf{m}\left(\mathbb{R}^{d} \times[0,1]\right)=E\left(R_{2}-R_{1}\right)=E_{v} R_{1} \leq c v(V)<\infty$. This implies that $\mathbf{X}$ is positive recurrent.

The invariant measure $m$ of the original process $X$ is the projection onto the first coordinate of $\mathbf{m}$. In particular, $X$ is also positive Harris recurrent, and $m$ can be represented as

$$
m(f)=E \int_{R_{1}}^{R_{2}} f\left(X_{S}\right) \mathrm{d} s .
$$


The ergodic theorem is then simply a consequence of the positive Harris property of $X$. Finally, the fact that $\Phi \circ V \in$ $L^{1}(m)$ is an immediate consequence of (2.12), based on Dynkin's formula.

Proof of Theorem 2.13. Theorem 2.13 follows from Theorem 5.2 of Löcherbach and Loukianova (2013) in [8] together with Proposition 3.6.

We finally proceed to the proof of Proposition 2.14.

Proof of Proposition 2.14. Let $X$ and $Y$ be a copies of the process, issued from $x$ (from $y$ respectively) at time 0 . Let $R_{1}$ and $R_{1}^{\prime}$ be the respective regeneration times. Using the same realization $V_{k}$ for $X$ and for $Y$ (recall (3.2)), it is clear that $R_{1}$ and $R_{1}^{\prime}$ are shift-coupling epochs for $X$ and for $Y$, i.e. $X_{R_{1}+}=Y_{R_{1}^{\prime}+.}$. If follows then from Thorisson [14] (1994), see also Roberts and Rosenthal [13] (1996), Proposition 5, that

$$
\left\|\frac{1}{t} \int_{0}^{t} P_{s}(x, \cdot) \mathrm{d} s-\frac{1}{t} \int_{0}^{t} P_{s}(y, \cdot) \mathrm{d} s\right\|_{\mathrm{TV}} \leq C \frac{1}{t}\left(E_{x}\left(R_{1}\right)+E_{y}\left(R_{1}^{\prime}\right)\right) .
$$

Recall that $p=1 /(1-\alpha)$. Then

$$
E_{x}\left(R_{1}\right) \leq\left(E_{x} R_{1}^{p}\right)^{1 / p} \leq c(V(x))^{(1-\alpha)} .
$$

Now, if $\alpha \geq \frac{1}{2}$, then $1-\alpha \leq \alpha$ and therefore,

$$
E_{x}\left(R_{1}\right) \leq c \Phi \circ V(x) \in L^{1}(m)
$$

In this case, we can integrate (3.30) against $m(\mathrm{~d} y)$ and obtain the second part of the assertion.

\section{Discussing the Lyapunov condition}

In this section, we discuss in an informal way several easily verifiable sufficient conditions implying Assumption 2.10 with $\Phi(v)=c v^{\alpha}, 0 \leq \alpha<1$. These conditions will involve different coefficients of the process. Recall that the infinitesimal generator $L$ of the process $X$ is given for every $\mathcal{C}^{2}$-function $\psi$ with compact support on $\mathbb{R}^{d}$ by

$$
L \psi(x)=\frac{1}{2} \sum_{i, j} a_{i j}(x) \frac{\partial^{2}}{\partial x_{i} \partial x_{j}} \psi(x)+g(x) \nabla \psi(x)+\int_{\mathbb{R}^{d}}[\psi(x+c(z, x))-\psi(x)] \gamma(z, x) \mu(\mathrm{d} z),
$$

where $a=\sigma \sigma^{*}$. In order to grant Assumption 2.10, we are seeking for conditions implying that

$$
L V \leq-c V^{\alpha}(x)+b 1_{C^{\prime}}(x),
$$

for some $0 \leq \alpha<1, b, c>0$. Recall that $C^{\prime}=\left\{x:\left|x-x_{0}\right|<\frac{\eta}{2}\right\}$ has been introduced in (2.10) above; the radius $\eta$ comes from the local Doeblin condition established in Proposition 2.6.

Example 4.1. If we choose for instance $V(x)=\left|x-x_{0}\right|^{2}$ and $\alpha=\frac{1}{2}$ it suffices to impose that for all $x \in \mathbb{R}^{d} \backslash C^{\prime}$,

$$
\operatorname{Tr}\left(\sigma \sigma^{*}\right)+2\left|g(x), x-x_{0}\right\rangle+\int_{\mathbb{R}^{d}}\left\langle 2\left(x-x_{0}\right)+c(z, x), c(z, x)|\gamma(z, x) \mu(\mathrm{d} z) \leq-c| x-x_{0}\right|
$$

and

$$
\sup _{x \in C^{\prime}} \int_{\mathbb{R}^{d}}\left\langle 2\left(x-x_{0}\right)+c(z, x), c(z, x)\right| \gamma(z, x) \mu(\mathrm{d} z)<+\infty .
$$


We now discuss several concrete sufficient conditions implying (4.1). In this context, it is interesting to notice that the influence of the different coefficients can be quite different. Some coefficients can work in a favorable way in order to ensure (4.1). In that case we will say that they are 'pushing' the diffusion into the set $C^{\prime}$. Other coefficients might play a neutral role or even work against (4.1). Since we have three natural parts of coefficients (diffusion part, drift and the jump part), we will discuss here the following cases: 'pushing' with the jumps only, 'pushing' with jumps and drift together and 'pushing' with the drift only.

\section{Pushing with the jumps}

We choose $V(x)=\left|x-x_{0}\right|^{2}+1$ and will impose both global and local conditions with respect to $z$.

1. Global condition with respect to $z . \forall z \in \mathbb{R}^{d}, \forall x \in \mathbb{R}^{d} \backslash C^{\prime}$,

$$
\left\langle c(z, x)+2\left(x-x_{0}\right), c(z, x)\right\rangle \leq 0 .
$$

Moreover we impose that

$$
\sup _{x \in C^{\prime}} \int_{E} \mid\left\langle c(z, x)+2\left(x-x_{0}\right), c(z, x)|| \gamma(z, x) \mu(\mathrm{d} z)<\infty .\right.
$$

2. Local conditions with respect to $z$ on some set $\mathcal{K}$. There exists a set $\mathcal{K}$ such that the following holds.

1. there exists $\xi>0$ such that for all $x \in \mathbb{R}^{d} \backslash C^{\prime}$,

$$
\int_{\mathcal{K}}|c(z, x)| \gamma(z, x) \mu(\mathrm{d} z)>\xi
$$

2. There exists $\zeta \in(0,1]$ such that for all $z \in \mathcal{K}$ and for all $x \in \mathbb{R}^{d} \backslash C^{\prime}$

$$
\left\langle c(z, x)+2\left(x-x_{0}\right), c(z, x)\right\rangle \leq-\zeta\left|c(z, x)+2\left(x-x_{0}\right)\right||c(z, x)| .
$$

3. For all $z \in \mathcal{K}$ and for all $x \in \mathbb{R}^{d} \backslash C^{\prime}$,

$$
|c(z, x)| \leq\left|x-x_{0}\right| \text {. }
$$

Notice that this last condition implies in particular that $\left|c(z, x)+2\left(x-x_{0}\right)\right| \geq\left|x-x_{0}\right|$.

Let us write $J V$ for the jump part of the infinitesimal generator of the process $X$, i.e. $J V(x)=\int_{E}(V(x+c(z, x))-$ $V(x)) \gamma(z, x) \mu(\mathrm{d} z)$. Then under the above conditions (4.4)-(4.8), for all $x \in \mathbb{R}^{d} \backslash C^{\prime}$,

$$
\begin{aligned}
J V(x) & =\int_{E}(V(x+c(z, x))-V(x)) \gamma(z, x) \mu(\mathrm{d} z) \\
& =\int_{E}\left\langle c(z, x)+2\left(x-x_{0}\right), c(z, x)\right| \gamma(z, x) \mu(\mathrm{d} z) \\
& \leq-\zeta \int_{\mathcal{K}}\left|c(z, x)+2\left(x-x_{0}\right)\right||c(z, x)| \gamma(z, x) \mu(\mathrm{d} z) \\
& \leq-\zeta\left|x-x_{0}\right| \int_{\mathcal{K}}|c(z, x)| \gamma(z, x) \mu(\mathrm{d} z) \\
& \leq-\zeta\left|x-x_{0}\right| \xi=-c(V(x)-1)^{\frac{1}{2}}=-\Phi \circ V(x)
\end{aligned}
$$

with $c=\zeta \xi>0$ and $\Phi(v)=c(v-1)^{\frac{1}{2}}$.

\section{Remark 4.2.}

1. Using the Cauchy-Schwarz inequality, (4.4) implies that for all $z \in \mathbb{R}^{d}$ and for all $x \in \mathbb{R}^{d} \backslash C^{\prime},|c(z, x)| \leq 2\left|x-x_{0}\right|$. In particular for all $x \in \mathbb{R}^{d} \backslash C^{\prime}, \sup _{z \in \mathbb{R}^{d}}|c(z, x)|<+\infty$. 
2. Condition (4.8) is a condition on the size of jumps ensuring that, at least for choices of $z$ belonging to $\mathcal{K}$, jumps are bounded from above by the distance to the point $x_{0}$. This implies that for starting points $x$ close to $C^{\prime}$, there is a possibility for the process to jump into $C^{\prime}$.

3. There is a simple geometric interpretation of the conditions (4.7) and (4.8). Indeed, they lead to the (effective) condition

$$
\left\langle c(z, x)+2\left(x-x_{0}\right), c(z, x)|\leq-\zeta| x-x_{0}|| c(z, x)\right|
$$

or

$$
2\left\langle\left(x-x_{0}\right), c(z, x)\right\rangle+|c(z, x)|^{2} \leq-\zeta\left|x-x_{0}\right||c(z, x)| .
$$

On the one hand, this implies that $\left\langle\left(x-x_{0}\right), c(z, x)\right\rangle \leq-\frac{\zeta}{2}\left|x-x_{0}\right||c(z, x)|$, which means that $c(z, x)$ belongs to the convex cone of direction $\left(x_{0}-x\right)$ and angle $\arccos \left(\frac{\zeta}{2}\right)$. On the other hand, using (4.8), the following condition

$$
2\left\langle\left(x-x_{0}\right), c(z, x)\right\rangle+|c(z, x)|\left|x-x_{0}\right| \leq-\zeta\left|x-x_{0}\right||c(z, x)|
$$

is a sufficient (but not necessary) condition which leads to $\left\langle\left(x-x_{0}\right), c(z, x)\right\rangle \leq-\frac{(1+\zeta)}{2}\left|x-x_{0}\right||c(z, x)|$. In other words, it suffices that $c(z, x)$ belongs to the convex cone of direction $\left(x_{0}-x\right)$ and angle $\arccos \left(\frac{(1+\zeta)}{2}\right)$.

In (4.9) above, we have only achieved a control on the jump part of the infinitesimal generator $L V$ of the process $X$. We impose the following additional conditions on the diffusion coefficient $\sigma$ and on the drift vector $g$ implying that the continuous part $A V(x)=\frac{1}{2} \sum_{1 \leq i, j \leq d} a_{i j}(x) \frac{\partial^{2} V(x)}{\partial x_{i} \partial x_{j}}+g(x) \nabla V(x)$ is negligible with respect to the jump part $J V(x)$.

We suppose

$$
\sup _{x \in \mathbb{R}^{d}} \operatorname{Tr}\left(\sigma \sigma^{*}(x)\right)<\infty \quad \text { and } \quad \Sigma=\sup _{x \in \mathbb{R}^{d}:\left|x-x_{0}\right| \geq \eta / 2} \frac{\left|\operatorname{Tr}\left(\sigma(x) \sigma^{*}(x)\right)\right|}{\left|x-x_{0}\right|}<\infty .
$$

Moreover, we suppose that

$$
\zeta \xi>\Sigma .
$$

Finally, concerning the drift part, we assume that

$$
\left\langle x-x_{0}, g(x)\right\rangle<0 \quad \text { for all } x \in \mathbb{R}^{d} \backslash C^{\prime} .
$$

Proposition 4.3. Under conditions (4.4)-(4.8), (4.10), (4.11) and (4.12), for $V(x)=\left|x-x_{0}\right|^{2}+1$, we have

$$
L V(x) \leq-c[V(x)-1]^{\frac{1}{2}},
$$

for all $x \in \mathbb{R}^{d} \backslash C^{\prime}$, where $c=\zeta \xi-\Sigma$. Moreover, $\sup _{x \in C^{\prime}}|L V(x)|<\infty$.

\section{Pushing with both jumps and drift part}

The conditions we made on the jump mechanism in the above paragraph are of course very strong. In this paragraph, we will therefore consider that these conditions hold only for $x$ belonging to some set $E_{1}$. Moreover, we will suppose that the drift coefficient contributes to force the diffusion into $C^{\prime}$ when $x$ belongs to another set $E_{2}$.

More precisely, we suppose that $E_{1} \subset \mathbb{R}^{d} \backslash C^{\prime}$ and take $E_{2}$ such that $E_{1} \cup E_{2}=\mathbb{R}^{d} \backslash C^{\prime}$.

We will impose the global condition (4.4) and (4.5), but we aim to weaken the conditions (4.6), (4.7) and (4.8) and impose them only for $x \in E_{1}$. For $x \in E_{2}$, we assume additionally that

$$
\operatorname{Tr}\left(\sigma \sigma^{*}\right)+2\left|g(x), x-x_{0}\right\rangle \leq-c\left|x-x_{0}\right| .
$$


Finally, we suppose that

$$
\sup _{x \in \mathbb{R}^{d}} \operatorname{Tr}\left(\sigma \sigma^{*}(x)\right)<\infty, \quad \text { that } \Sigma=\sup _{x \in E_{1}} \frac{\left|\operatorname{Tr}\left(\sigma(x) \sigma^{*}(x)\right)\right|}{\left|x-x_{0}\right|}<\infty,
$$

that (4.11) holds and

$$
\left\langle x-x_{0}, g(x)\right\rangle<0 \quad \text { for all } x \in E_{1} .
$$

Then under the above conditions,

$$
L V(x) \leq-c[V(x)-1]^{\frac{1}{2}},
$$

for all $x \in \mathbb{R}^{d} \backslash C^{\prime}$, and $\sup _{x \in C^{\prime}}|L V(x)|<\infty$.

Example 4.4. We continue Example 2.9 item 1. and take $x_{0}=0$. We suppose additionally that for all $x \in \mathbb{R} \backslash C^{\prime}$, $|f(x)| \leq|x|$ and $\operatorname{sgn}(f(x))=-\operatorname{sgn}(x)$. Finally, we impose (4.10) and (4.12).

Put $E_{1}=\left[-M-\frac{\eta}{2}\right] \cup\left[\frac{\eta}{2}+M\right]$ and choose $\mathcal{K}=[a, a+2 R]$ in such a way that $\int_{\mathcal{K}}\left[1-e^{-|z|}\right] \mathrm{d} z=\frac{1}{2}$. It follows from our assumptions on $\gamma$ and $c$ that for all $(z, x) \in \mathcal{K} \times E_{1}$

$$
\gamma(z, x) \geq \underline{\gamma}>0 \quad \text { and } \quad f(x) \geq \underline{f}>0 .
$$

Moreover, we assume that

$$
\underline{f} \cdot \underline{\gamma}>2 \frac{\Sigma}{\zeta}
$$

It is clear that (4.6) is verified for all $x \in E_{1}$, and, moreover, that the jumps are strong enough to ensure the drift condition even in presence of the Brownian part.

Since $|f(x)| \leq|x|$ for all $x \notin C^{\prime}$, (4.8) is satisfied. Finally (4.4) follows from the fact that $\operatorname{sgn}(f(x))=-\operatorname{sgn}(x)$, for all $x \notin C^{\prime}$, and (4.7) follows with $\zeta=1$.

Pushing only with the drift

In order to exploit the effect of a drift vector, it might be suitable to consider a different Lyapunov function as we are going to explain in the present section. Let for example $x_{0}=0$ and $V(x)=|x|+1=\sqrt{x_{1}^{2}+\cdots+x_{d}^{2}}+1$. Then

$$
\nabla V(x)=\frac{x}{|x|}, \quad \frac{\partial^{2}}{\partial_{i} \partial_{j}} V(x)=\frac{\delta_{i j}}{|x|}-\frac{x_{i} x_{j}}{|x|^{3}}
$$

Due to Assumption 2.1 item 4., we know that

$$
\sup _{x} \int_{E}|c(z, x)| \gamma(z, x) \mu(\mathrm{d} z)=: D<\infty .
$$

We suppose moreover that $\sigma$ is bounded and take $D$ sufficiently large such that $\left|a_{i j}\right|<D$, where $a=\sigma \sigma^{*}$.

We denote by $|x|_{1}=\left|x_{1}\right|+\cdots+\left|x_{d}\right|$ the $L^{1}$-norm on $\mathbb{R}^{d}$ and use that $\frac{1}{\sqrt{d}}|x|_{1} \leq|x|$. We impose the following drift condition on $g$. For every $x \notin C^{\prime}$,

$$
\langle x, g(x)\rangle \leq-c|x|^{1+\alpha}-D|x|-d D,
$$


for some $0 \leq \alpha<1$. Then it is immediate to see that for all $x \notin C^{\prime}$,

$$
\begin{aligned}
L V(x) & \leq \frac{D}{2}\left(\frac{d}{|x|}+\sum_{1 \leq i, j \leq d} \frac{\left|x_{i}\right|\left|x_{j}\right|}{|x|^{3}}\right)+\frac{\langle x, g(x)\rangle}{|x|}+\underbrace{\int_{E}|c(z, x)| \gamma(z, x) \mu(\mathrm{d} z)}_{\leq D} \\
& =\frac{D}{2}\left(\frac{d}{|x|}+\frac{|x|_{1}^{2}}{|x|^{3}}\right)+\frac{\langle x, g(x)\rangle}{|x|}+D \\
& \leq \frac{d D}{|x|}+\frac{\langle x, g(x)\rangle}{|x|}+D \\
& \leq \frac{d D}{|x|}-\frac{1}{|x|}\left(c|x|^{1+\alpha}+D|x|+d D\right)+D \leq-c|x|^{\alpha} .
\end{aligned}
$$

\section{Proofs}

In the following, $B(x, r)=\left\{y \in \mathbb{R}^{d}:|y-x|<r\right\}$ denotes the ball with radius $r$ and center $x \in \mathbb{R}^{d}$. Moreover, for $B \in \mathbb{R}^{d \times d \times d}$, we shall write $\|B\|$ for the associated operator norm ; we will also denote by $\operatorname{dg}(a)$ the differential of a function $g$ at $a$. The main ingredient of the proof of Proposition 2.6 is the following result.

Lemma 5.1. Let $\Psi_{x}(z)=x+c(z, x), \mathcal{K}=\left\{z:\left|z-z_{0}\right| \leq R\right\}, \Psi_{x}(\mathcal{K})=\left\{\Psi_{x}(z), z \in \mathcal{K}\right\}$ and $a_{x}=x+c\left(z_{0}, x\right)=$ $\Psi_{x}\left(z_{0}\right)$. Put

$$
\rho=\frac{A}{2}\left(R \wedge \frac{1}{2 K}\right)
$$

Then there exists $\eta>0$ such that

$$
B\left(a_{x_{0}}, \frac{\rho}{2}\right) \subset \bigcap_{x \in B\left(x_{0}, \eta\right)} \Psi_{x}(\mathcal{K}) .
$$

Moreover, for all $x$ such that $\left|x-x_{0}\right| \leq r$, we have $B\left(a_{x}, \rho\right) \subset \Psi_{x}(\mathcal{K})$, and there exists $\mathcal{K}_{x} \subset \mathcal{K}$ such that $z \mapsto \Psi_{x}(z)$ is a $\mathcal{C}^{1}$-diffeomorphism from $\mathcal{K}_{x}$ to $B\left(a_{x}, \rho\right)$.

Remark 5.2. The ball $B$ appearing in (2.9) can be chosen as $B=B\left(a_{x_{0}}, \rho / 2\right)$ with $\rho$ as in (5.1) and $a_{x_{0}}=x_{0}+$ $c\left(z_{0}, x_{0}\right)$.

We will first admit this lemma and show how we can use it to prove Proposition 2.6.

Proof of Proposition 2.6. We admit Lemma 5.1 and take $\mathcal{K}=\left\{z:\left|z-z_{0}\right| \leq R\right\}$ as there. As a consequence, there exists a ball $B\left(x_{0}, \eta\right)$ such that for all $x \in B\left(x_{0}, \eta\right), B\left(a_{x_{0}}, \frac{\rho}{2}\right) \subset \Psi_{x}(\mathcal{K})$. Choose $\mathcal{K}^{\prime \prime} \subset \mathcal{K}$ such that $\Psi_{x}: \mathcal{K}^{\prime \prime} \rightarrow$ $B\left(a_{x_{0}}, \frac{\rho}{2}\right)$ is a $\mathcal{C}^{1}$-diffeomorphism for all $x \in B\left(x_{0}, \eta\right){ }^{2}$ Since for all $(z, x) \in \mathcal{K} \times B\left(x_{0}, \eta\right), \gamma(z, x) h(z) \geq \varepsilon$, we now have

$$
\int_{E_{n}} 1_{V}\left(\psi_{x}(z)\right) \gamma(z, x) \mathrm{d} \mu(z) \geq \varepsilon \int_{K^{\prime \prime}} 1_{V}\left(\psi_{x}(z)\right) \mathrm{d} z=\varepsilon \int_{B\left(a_{x_{0}}, \frac{\rho}{2}\right)} 1_{V}(y)\left|J_{\psi_{x}^{-1}}(y)\right| \mathrm{d} y .
$$

Put $z=\psi_{x}^{-1}(y)$, then

$$
\left|J_{\psi_{x}^{-1}}(y)\right|=\frac{1}{\left|J_{\psi_{x}}(z)\right|}=\frac{1}{\left|\operatorname{det}\left(\nabla_{z} c(z, x)\right)\right|}
$$

\footnotetext{
${ }^{2}$ Indeed, from Lemma 5.1, there exists $\mathcal{K}^{\prime} \subset \mathcal{K}$ such that $\Psi_{x}: \mathcal{K}^{\prime} \rightarrow B\left(a_{x}, \rho\right)$ is a $\mathcal{C}^{1}$-diffeomorphism, and since $B\left(a_{x_{0}}, \frac{\rho}{2}\right) \subset B\left(a_{x}, \rho\right)$, there exists $\mathcal{K}^{\prime \prime} \subset \mathcal{K}^{\prime} \subset \mathcal{K}$ such that $\Psi_{x}: \mathcal{K}^{\prime \prime} \rightarrow B\left(a_{x_{0}}, \frac{\rho}{2}\right)$ is a $\mathcal{C}^{1}$-diffeomorphism.
} 
and, using Hadamard's Inequality,

$$
\left|\operatorname{det}\left(\nabla_{z} c(z, x)\right)\right| \leq \prod_{i=1}^{d}\left|\partial_{z_{i}} c(z, x)\right| .
$$

As a consequence, we obtain

$$
\int_{E_{n}} 1_{V}\left(\psi_{x}(z)\right) \gamma(z, x) \mathrm{d} \mu(z) \geq \frac{\varepsilon}{S^{d}} \lambda\left(V \cap B\left(a_{x_{0}}, \frac{\rho}{2}\right)\right)
$$

which, together with (2.5), ends the proof.

It remains to give a proof of Lemma 5.1. This proof goes through several intermediate steps which are given now.

Lemma 5.3. Let $g: \mathbb{R}^{d} \rightarrow \mathbb{R}^{d}$ be a $\mathcal{C}^{2}$-function such that

1. $g(0)=0$,

2. $(\mathrm{d} g)(0)=\mathrm{Id}$,

3. there exist $R, K>0$ such that for all $z \in B(0, R)$,

$$
\sum_{i, j, k}\left|\frac{\partial^{2} g_{k}}{\partial z_{i} \partial z_{j}}(z)\right| \leq K
$$

Put $\tilde{R}=R \wedge \frac{1}{2 K}$. Then $B\left(0, \frac{\tilde{R}}{2}\right) \subset g(B(0, \tilde{R}))$.

Proof. The third condition allows to apply the Mean Value Inequality to $z \mapsto(\mathrm{d} g)(z)$ since

$$
\|d(\mathrm{~d} g)(z)\| \mid \leq K, \quad \forall z \in B(0, R) .
$$

Therefore, with $\tilde{R}=R \wedge \frac{1}{2 K}$,

$$
\|\mathrm{d} g(z)-\mathrm{Id}\|=\|\mathrm{d} g(z)-\mathrm{d} g(0)\| \leq K|z| \leq \frac{1}{2}, \quad \forall z \in B(0, \tilde{R}) .
$$

Let now $y \in B\left(0, \frac{\tilde{R}}{2}\right)$ and set $h: \overline{B(0, \tilde{R})} \rightarrow \mathbb{R}^{d}, z \mapsto h(z):=y+z-g(z)$. We have

$$
\|\mathrm{d} h(z)\|=\|\operatorname{Id}-\mathrm{d} g(z)\| \leq \frac{1}{2}, \quad \forall z \in B(0, \tilde{R}) .
$$

Using again the Mean Value Inequality, we obtain for all $z, z^{\prime} \in \overline{B(0, \tilde{R})}$,

$$
\left|h(z)-h\left(z^{\prime}\right)\right| \leq \frac{1}{2}\left|z-z^{\prime}\right| .
$$

In particular $|h(z)| \leq \frac{1}{2}\left|z-z^{\prime}\right|+\left|h\left(z^{\prime}\right)\right|$, so $|h(z)| \leq \frac{1}{2}|z|+|h(0)|=\frac{1}{2}|z|+|y|<\tilde{R}$, for all $z \in \overline{B(0, \tilde{R})}$.

This last result highlights two facts. First, $h$ is an $\frac{1}{2}$-contraction from the complete space $\overline{B(0, \tilde{R})}$ into itself, so the fixed-point theorem gives us the existence of $z \in \overline{B(0, \tilde{R})}$ such that $h(z)=z$, and, secondly, the range of $h$ defined on $\overline{B(0, \tilde{R})}$ is $B(0, \tilde{R})$. So we have in fact the existence of $z \in B(0, \tilde{R})$ such that $h(z)=z$, or equivalently, $g(z)=y$, which ends the proof.

\section{Remark 5.4.}

1. $g$ is in fact a $\mathcal{C}^{1}$-diffeomorphism from $V=B(0, \tilde{R}) \cap g^{-1}\left(B\left(0, \frac{\tilde{R}}{2}\right)\right)$ to $B\left(0, \frac{\tilde{R}}{2}\right)$.

2. We could have chosen $\tilde{R}=R \wedge \frac{1-\varepsilon^{\prime}}{K}$ for any $\left.\varepsilon^{\prime} \in\right] 0,1[$. 
Lemma 5.5. Let $A$ be a $d \times d$ matrix such that

$\forall y \in \mathbb{R}^{d}, \quad|A y| \geq K|y|$.

Then

$$
B(A u, K \tilde{R}) \subset A(B(u, \tilde{R})) .
$$

Proof. Notice first that $A$ is clearly invertible. Let now $y \in B(A u, K \tilde{R})$. Then for $v \in \mathbb{R}^{d}$,

$$
|v|=\left|A\left(A^{-1} v\right)\right| \geq K\left|A^{-1} v\right|,
$$

so, with $v=y-A u$,

$$
K \tilde{R} \geq|y-A u| \geq K\left|A^{-1}(y-A u)\right|=K\left|A^{-1} y-u\right|,
$$

or, equivalently, $\tilde{R} \geq\left|A^{-1} y-u\right|$ implying that $A^{-1} y \in B(u, \tilde{R})$ and $y \in A(B(u, \tilde{R}))$.

We now have the following extension of Lemma 5.3.

Proposition 5.6. Let $f: \mathbb{R}^{d} \rightarrow \mathbb{R}^{d}$ a $\mathcal{C}^{2}$-function and $a \in \mathbb{R}^{d}$ such that

1. $|\mathrm{d} f(a) y| \geq A|y|$ for all $y \in \mathbb{R}^{d}$,

2. there exist $R, K>0$ such that for all $y \in B(a, R)$,

$$
\left\|(\mathrm{d} f(a))^{-1}\right\| \sum_{i, j}\left|\frac{\partial^{2} f}{\partial z_{i} \partial z_{j}}(y)\right| \leq \frac{K}{d} .
$$

Then, with $\tilde{R}=R \wedge \frac{1}{2 K}$,

$$
B\left(f(a), A \frac{\tilde{R}}{2}\right) \subset f(B(a, \tilde{R})) .
$$

Proof. (1) We use Lemma 5.3 with

$$
g(z)=(\mathrm{d} f(a))^{-1}(f(a+z)-f(a)) .
$$

All hypotheses needed in Lemma 5.3 are satisfied since

$$
\frac{\partial^{2} g}{\partial z_{i} \partial z_{j}}(z)=(\mathrm{d} f(a))^{-1} \frac{\partial^{2} f}{\partial z_{i} \partial z_{j}}(a+z) .
$$

Thus

$$
B\left(0, \frac{\tilde{R}}{2}\right) \subset g(B(0, \tilde{R})) .
$$

(2) Since $f(y)=\mathrm{d} f(a) g(y-a)+f(a)$, using Lemma 5.5,

$$
B\left(0, A \frac{\tilde{R}}{2}\right) \subset \mathrm{d} f(a)\left(B\left(0, \frac{\tilde{R}}{2}\right)\right) \subset \mathrm{d} f(a) g(B(0, \tilde{R})),
$$

where we have used the preceding step in order to obtain the last inclusion. Therefore,

$$
B\left(f(a), A \frac{\tilde{R}}{2}\right) \subset f(B(a, \tilde{R})) .
$$


We are now able to prove Lemma 5.1.

Proof of Lemma 5.1. (1) Let $x \in \overline{B\left(x_{0}, r\right)}$. We can apply Proposition 5.6 with $a=z_{0}, f=\Psi_{x}$ which gives $\rho=$ $\frac{A}{2}\left(R \wedge \frac{1}{2 K}\right)$ such that

$$
B\left(a_{x}, \rho\right) \subset \Psi_{x}\left(B\left(z_{0}, \frac{2 \rho}{A}\right)\right) \subset \Psi_{x}(\mathcal{K}),
$$

where we recall that $\mathcal{K}=\overline{B\left(z_{0}, R\right)}$. Since our conditions are uniform in $x$, the radius $\rho$ will be the same for all $x \in \overline{B\left(x_{0}, r\right)}$.

(2) The previous point implies in particular that

$$
B\left(a_{x_{0}}, \rho\right) \subset \Psi_{x_{0}}(\mathcal{K}) .
$$

Since $x \mapsto \Psi_{x}\left(z_{0}\right)$ is continuous, there exists $\eta$ with $r>\eta>0$ such that

$$
\left|x-x_{0}\right|<\eta \Longrightarrow\left|\Psi_{x}\left(z_{0}\right)-\Psi_{x_{0}}\left(z_{0}\right)\right|<\frac{\rho}{2} .
$$

Therefore,

$$
\bigcap_{y \in B\left(x_{0}, \eta\right)} B\left(a_{y}, \rho\right) \subset \Psi_{x}(\mathcal{K}),
$$

so it is sufficient to prove that

$$
B\left(a_{x_{0}}, \frac{\rho}{2}\right) \subset \bigcap_{y \in B\left(x_{0}, \eta\right)} B\left(a_{y}, \rho\right)
$$

which can be seen as follows. Let $y \in B\left(a_{x_{0}}, \frac{\rho}{2}\right)$ and $x \in B\left(x_{0}, \eta\right)$, then

$$
\begin{aligned}
\left|a_{x}-y\right| & \leq\left|a_{x_{0}}-y\right|+\left|a_{x}-a_{x_{0}}\right| \\
& =\left|a_{x_{0}}-y\right|+\left|\Psi_{x}\left(z_{0}\right)-\Psi_{x_{0}}\left(z_{0}\right)\right| \\
& <\frac{\rho}{2}+\frac{\rho}{2}=\rho,
\end{aligned}
$$

so $y \in B\left(a_{x}, \rho\right)$, for every $x \in B\left(x_{0}, \eta\right)$ and the statement is proved.

Proofs of Remarks 2.7 and 5.2. Recall that we have imposed the additional hypothesis $L_{c}=\sup _{z \in \mathcal{K}} L_{c}(z)<\infty$. Since

$$
|c(z, x)-c(z, y)| \leq L_{c}(z)|x-y|, \quad \forall x, y \in \mathbb{R}^{d}, \forall z \in E,
$$

it is sufficient to set

$$
\eta=\frac{\rho}{2\left(1+L_{c}\right)} \wedge r
$$

in order to grant (5.4).

We finish this section showing that the growth-fragmentation model introduced in Section 2 satisfies our assumptions.

Proof of Example 2.8. We show that Example 2.8 satisfies our Assumptions 2.1-2.4.

Indeed, items 1. and 2. of Assumption 2.1 follow immediately. 
In order to check points 3. and 4. of Assumption 2.1, the main point is that for any fixed $x$ and for small $z$, $\gamma(z, x)|c(z, x)|=f(x) \psi(x) \frac{1}{z}[1-\kappa(z)] \sim f(x) \psi(x) \kappa^{\prime}(0)$, which is integrable in $z \in[0, \delta]$, for any $\delta>0$. Assumption 2.2 holds on $\left\{x \geq 0:\left|x-x_{0}\right|<r\right\}$ for any $x_{0} \geq 0$ and $r>0$, since $f(x)>0$ for any $x$. We are now going to check Assumption 2.4. In order to check (2.6), note that

$$
\partial_{z} c\left(z_{0}, x\right)=\psi(x) \kappa^{\prime}\left(z_{0}\right),
$$

implying that (2.6) holds with

$$
A=\kappa^{\prime}\left(z_{0}\right) \inf _{x:\left|x-x_{0}\right|<r} \psi(x) \geq \kappa^{\prime}\left(z_{0}\right) a .
$$

Moreover, (2.7) holds due to the non-degeneracy of $\kappa^{\prime}(z) \neq 0$ for all $z>0$, with a constant $K$ only depending on $z_{0}$ and on $R$, but not on $x_{0}$, nor on $r$. Finally, (2.8) trivially holds. $\psi$ being 1-Lipschitz, we have that $L_{c}(z)=\kappa(z)$, and therefore $L_{c}=\sup _{z:\left|z-z_{0}\right|<R} L_{c}(z)=\sup _{z:\left|z-z_{0}\right|<R} \kappa(z)$, implying that the radius $\eta$ given in (2.11) can be chosen as

$$
\eta=C\left(z_{0}, R\right) a \wedge r
$$

where $C\left(z_{0}, R\right)$ is a constant depending on $K$ and on $L_{c}$, but not on $x_{0}$ nor on $r$.

Finally, we give the proof of (2.23): Indeed,

$$
L V(x)=2 x+\int_{0}^{\infty} e^{-z}[2 x+c(z, x)] c(z, x) \gamma(z, x) \mathrm{d} z
$$

with $c(z, x)=(\kappa(z)-1) \psi(x)$ and $\gamma(z, x)=f(x) \frac{1}{z}$. But for $x>\eta / 2$, using that $(\kappa(z)-1)^{2} \leq|\kappa(z)-1|$, since $|\kappa(z)-1| \leq 1$,

$$
\begin{aligned}
{[2 x+c(z, x)] c(z, x) } & =2 x(\kappa(z)-1) \psi(x)+(\kappa(z)-1)^{2} \psi^{2}(x) \\
& \leq-2 x|\kappa(z)-1| \psi(x)+|\kappa(z)-1| \psi(x) x \\
& =-\psi(\eta / 2) x|\kappa(z)-1|,
\end{aligned}
$$

since $\psi(\eta / 2) \leq \psi(x) \leq x$ for all $x \geq \eta / 2$.

As a consequence,

$$
L V(x) \leq-x\left[\psi\left(\frac{\eta}{2}\right) \int_{0}^{\infty} \frac{1}{z}(1-\kappa(z)) e^{-z} \mathrm{~d} z-2\right]=-c x=-c(V(x)-1)^{1 / 2},
$$

where $c=\psi\left(\frac{\eta}{2}\right) \int_{0}^{\infty} \frac{1}{z}(1-\kappa(z)) e^{-z} \mathrm{~d} z-2>0$ by assumption (2.22).

\section{Acknowledgements}

We thank Vlad Bally for many nice discussions on the subject. We also thank two anonymous referees for helpful comments and suggestions.

This research has been conducted as part of the project Labex MME-DII (ANR11-LBX-0023-01).

\section{References}

[1] K. B. Athreya and P. Ney. A new approach to the limit theory of recurrent Markov chains. Trans. Amer. Math. Soc. 245 (1978) $493-501$. MR0511425

[2] J. Azéma, M. Duflo and D. Revuz. Mesure invariante des processus de Markov récurrents. Séminaire de probabilités (Strasbourg) 3 (1969) 24-33. MR0260014

[3] R. Douc, G. Fort and A. Guillin. Subgeometric rates of convergence of $f$-ergodic strong Markov processes. Stochastic Process. Appl. 119 (3) (2009) 897-923. MR2499863 
[4] J. Duan and H. Qiao. Stationary measures for stochastic differential equations with jumps. ArXiv, 2014.

[5] C. Graham. McKean-Vlasov Ito-Skorokhod equations, and nonlinear diffusions with discrete jump sets. Stochastic Process. Appl. 40 (1992) 69-82. MR1145460

[6] N. Krell. Statistical estimation of jump rates for a specific class of Piecewise Deterministic Markov Processes. ArXiv, 2015.

[7] A. M. Kulik. Exponential ergodicity of the solutions to SDE's with a jump noise. Stochastic Process. Appl. 119 (2) (2009) $602-632$. MR2494006

[8] E. Löcherbach and D. Loukianova. Polynomial deviation bounds for recurrent Harris processes having general state space. ESAIM Probab. Stat. 17 (2013) 195-218. doi:10.1051/ps/2011156. MR3021315

[9] H. Masuda. Ergodicity and exponential $\beta$-mixing bounds for multidimensional diffusions with jumps. Stochastic Process. Appl. 117 (2007) 35-56. MR2287102

[10] S. P. Meyn and R. L. Tweedie. Markov Chains and Stochastic Stability. Springer-Verlag, London, 1993. MR1287609

[11] S. P. Meyn and R. L. Tweedie. Stability of Markovian processes III : Foster-Lyapunov criteria for continuous-time processes. Adv. in Appl. Probab. 25 (3) (1993) 518-548. MR1234295

[12] E. Nummelin. A splitting technique for Harris recurrent Markov chains. Z. Wahrsch. Verw. Gebiete 43 (1978) 309-318. MR0501353

[13] G. O. Roberts and J. S. Rosenthal. Quantitative bounds for convergence rates of continuous time Markov processes. Electron. J. Probab. 1 (9) (1996) 1-21. MR1423462

[14] H. Thorisson The coupling method and regenerative processes. In Analysis, Algebra, and Computers in Mathematical Research. Proceedings of the 21st Nordic Congress of Mathematicians, Lulea Univ. of Technology, Sweden, 1992 347-363. M. Gyllenberg et al. (Eds). Lect. Notes Pure Appl. Math. 156. Marcel Dekker, New York, 1994. MR1280957

[15] L. Xu. Exponential mixing of 2D SDE's forced by degenerate Lévy noises. J. Evol. Equ. 14 (2) (2014) 249-272. doi:10.1007/s00028-0130212-4. MR3207614 